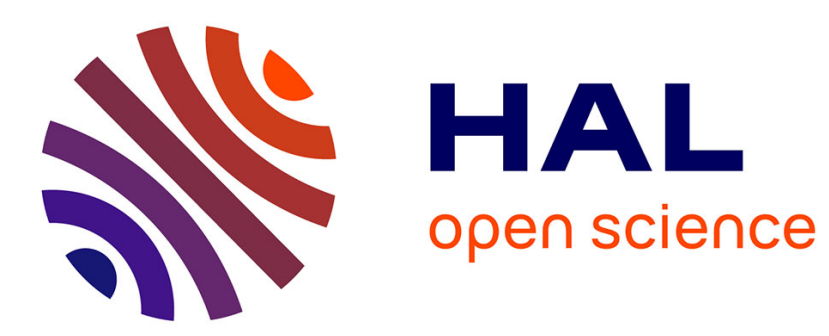

\title{
Le démantèlement méthodique et tragique des institutions grecques de santé publique
}

\author{
Noëlle Burgi
}

\section{To cite this version:}

Noëlle Burgi. Le démantèlement méthodique et tragique des institutions grecques de santé publique.

La Revue de l'IRES, 2018. hal-01967922

\section{HAL Id: hal-01967922 \\ https://hal.science/hal-01967922}

Submitted on 1 Jan 2019

HAL is a multi-disciplinary open access archive for the deposit and dissemination of scientific research documents, whether they are published or not. The documents may come from teaching and research institutions in France or abroad, or from public or private research centers.
L'archive ouverte pluridisciplinaire HAL, est destinée au dépôt et à la diffusion de documents scientifiques de niveau recherche, publiés ou non, émanant des établissements d'enseignement et de recherche français ou étrangers, des laboratoires publics ou privés. 


\title{
Le démantèlement méthodique et tragique des institutions grecques de santé publique
}

\author{
Noëlle BURGI ${ }^{1}$
}

Le système national de santé grec a été démantelé par l'application d'un ensemble de mesures imposées depuis 2010 par les créanciers de la Grèce dans les secteurs de santé primaire, secondaire et pharmaceutique. Ce texte présente une analyse critique des principales mesures de compression budgétaire mises en place dans ces secteurs et introduit un débat sur des initiatives communautaires censées renforcer certains déterminants sociaux de la santé (indemnités de chômage, assurance maladie, revenu minimum garanti). Il apparaît que les politiques mémorandaires ont manqué le but d'efficience et d'efficacité affiché, mais peut-être pas le projet implicite de construire un "nouveau modèle social européen " réduit à quelques prestations tout juste suffisantes à la survie des dépossédés. En s'appuyant sur de nombreux travaux scientifiques, des entretiens en Grèce auprès de militants et dans des établissements de soin et une enquête en cours dans des quartiers ouvriers $d u$ Pirée, l'article conclut à l'épuisement - passager ? - des forces luttant pour la survie des droits sociaux démocratiques.

Depuis 2010, la Grèce est soumise à un régime de discipline et de contrôle de ses finances et politiques publiques sans équivalent dans l'histoire européenne d'après 1945. Peu après la révélation en 2009 des « vrais » chiffres, jusque-là maquillés, du déficit public grec ${ }^{2}$ par le gouvernement tout juste élu de Georges Papandreou, la troikka (Commission européenne, Banque centrale européenne et Fonds monétaire international ${ }^{3}$ )

1. CNRS, CESSP.

2. Surestimé à $15,8 \%$ du PIB après de troublantes révisions à la hausse, mais tout de même bien supérieur à la limite des $3 \%$ autorisée par le traité de Maastricht. Pour une analyse approfondie, voir le site du Comité pour l'abolition des dettes illégitimes (CADTM) et en particulier le Rapport CADTM 2015

3. Rejointe par le Mécanisme européen de stabilité (MES) en 2015, elle est devenue un Quartet. 
des institutions créditrices du pays a mis le pays sous tutelle à travers une série de plans d'ajustement structurel ou mémorandums (2010, 2012, $2015^{4}$ ) censés résoudre le problème de sa dette et le remettre sur le chemin de la croissance. Les objectifs affichés de ces programmes n'ont pas été atteints, au contraire. Sept ans après leur mise en œuvre, la Grèce se trouve dans une situation bien pire qu'en 2010.

Ce n'est pas faute d'avoir appliqué les mesures contenues dans les mémorandums, ou d'avoir prolongé à souhait leur mise en œuvre. La troikka se dit régulièrement « impatiente » et reproche au gouvernement grec ses retards dans la mise en œuvre des «paquets » de réformes prescrites, mais elle ne lui laisse pratiquement aucune marge de manœuvre. En effet, l'approche générale des mémorandums est celle d'un "système de surveillance intense et quasi permanent ». Joaquin Almunia, alors commissaire européen chargé des Affaires économiques et monétaires, avait utilisé cette formule en février 2010 pour désigner le régime qui serait bientôt infligé à la Grèce : celle-ci devrait « quantifier » et « préciser » les mesures annoncées, « envoyer le calendrier » de leur mise en œuvre, rendre compte très régulièrement de l'état d'avancement de son programme et s'engager à prendre incessamment des mesures supplémentaires si ces objectifs n'étaient pas atteints ${ }^{5}$. "C'est la première fois que des instruments de surveillance économique et budgétaire sont utilisés simultanément et de manière intégrée », se félicitait pour sa part la Commission européenne. De fait, pour éviter tout retour en arrière et rendre les effets des programmes d'austérité irréversibles, la méthode appliquée en Grèce (puis à d'autres pays vulnérables) consiste à concentrer les mesures (frontloading) et à en vérifier constamment la bonne exécution, notamment à la veille des versements du prêt, prévus par tranches en principe tous les trimestres. L'état d'avancement du programme d'austérité est lui-même subordonné à l'appréciation qu'en donnent les experts et contrôleurs de la troïka, installés à plein temps dans les ministères et soutenus dans leur tâche par d'autres experts. Ce qui conduit les créanciers du pays à des actes répétitifs d'intimidation et de chantage. Ils exigent régulièrement des mesures d'austérité plus convaincantes à leurs yeux, reportent les versements, menacent de couper les vivres.

4. De très nombreux documents sont associés aux mémorandums (CADTM, 2015). Pour les deux premiers mémorandums, on peut consulter les documents de la Commission européenne (European Commission, 2010, 2012). Pour le troisième, on dispose d'une version commentée par Yanis Varoufakis, ex-ministre des Finances du premier gouvernement Tsipras, du Communiqué de l'Eurogroupe et du mémorandum (https://varoufakis.files.wordpress.com/2015/08/mou-annotated-by-yv.pdf) et de sa traduction française sur le blog de Paul Jorion (http://www.pauljorion. com/blog/2015/07/16/communique-sur-la-grece-avec-les-annotations-de-yanis-varoufakis/).

5. Joaquin Almunia est largement cité dans la presse quotidienne du 12 février 2010. Sur l'approche méthodique et punitive des mémorandums et, au-delà, de la « nouvelle gouvernance européenne » entérinée par le Traité budgétaire européen (2012), voir Greer et al. (2016) ; Stamati, Baeten (2014) ; Burgi (2014a, 2014b), ainsi que les mémorandums eux-mêmes (voir note précédente). 
Les mémorandums font partie d'une stratégie plus globale fondée sur une doctrine dite d'" austérité expansionniste » en vertu de laquelle une baisse des coûts relatifs par rapport à d'autres économies permet de créer les conditions d'une reprise par les exportations. En l'absence d'une dévaluation de la monnaie, elle suppose que soient appliquées des politiques austéritaires ${ }^{6}$ dites de dévaluation interne : coupes plus ou moins importantes dans la protection sociale, la santé, l'éducation et d'autres services publics, baisses des salaires et des retraites, augmentation des impôts indirects sur la consommation, dérégulation des relations professionnelles et du droit du travail... Cette doctrine a été discréditée sur les plans méthodologique et empirique (Jayadev, Konczal, 2010 ; FMI, 2012 ; Herndon et al., 2013) : comme l'avait noté Paul Krugman, ses prédictions « ont été entièrement contredites par la réalité, et la recherche académique invoquée pour soutenir [cette] position s'est révélée truffée d'erreurs, d'omissions et de statistiques suspectes ». Entre autres difficultés, la dévaluation interne ne peut pas fonctionner si tous les pays d'une même zone économique l'adoptent simultanément: dans ce cas, son seul effet est de conduire à une baisse des niveaux de vie et à réduire l'accès des populations aux biens publics essentiels. C'est néanmoins la voie qui fut choisie en réponse à la crise financière de 2007-2008 pour tous les États membres de l'Union économique et monétaire (UEM). En Grèce, les programmes d'austérité sans fin et toujours plus intenses ont entraîné une dépression économique et une récession sociale jamais vues en Europe en temps de paix (Ioakeimoglou, 2017). Persistantes, les institutions créditrices du pays lui appliquent encore ces mesures.

La société grecque a pourtant résisté au traitement subi. Le pays fut secoué par une impressionnante vague de grèves et de manifestations qui dura jusqu'en 2012 malgré une tout aussi impressionnante répression policière (Kotronaki, 2014). Le système politique se fractura. Tandis qu'aux élections de 2012 le Parti socialiste panhellénique (Pasok) s'effondrait et que les conservateurs de Nouvelle démocratie (ND), quoique plus résistants, s'affaiblissaient, le parti de la gauche radicale Syriza devenait le premier parti d'opposition. Emmené par Alexis Tsipras et porté par le mouvement social, il venait de connaittre une ascension fulgurante : tout juste entré au Parlement en 2009 avec 4,6\% des voix, il obtint $27 \%$ des suffrages en juin 2012. Sa montée se poursuivit jusqu'aux législatives de janvier 2015 qui le portèrent au pouvoir. Syriza incarnait l'espoir qu'un gouvernement formé par une nouvelle génération de politiciens de gauche, jeunes et non corrompus, rendrait sa dignité et sa souveraineté au pays et mettrait fin aux mémorandums. Cependant, n'ayant réussi à obtenir aucune concession pendant les six premiers mois de stériles négociations avec la troikka, Alexis

6. Au sens où ces politiques sont imposées par la contrainte (Lochard, Pernot, 2010). 
Tsipras organisa un référendum le 5 juillet 2015 à l'issue duquel les citoyens grecs dirent massivement «Non » (61,5\% des voix) à la poursuite de l'austérité. Le Premier ministre n'avait pas prévu ce résultat et ne pouvait ni ne voulait affronter ses créditeurs au point d'envisager une sortie de la Grèce de l'euro (le « Grexit»). Sept jours plus tard, il transformait le «Non» en " Oui » et cédait face à l'Union européenne. Il acceptait l'ultimatum du ministre des Finances allemand, Wolfgang Schäuble, en vertu duquel la Grèce renonçait à sa souveraineté et se pliait, en contrepartie d'un nouveau mémorandum (incluant un prêt de 86 milliards d'euros étalés sur trois ans et servant uniquement à rembourser la dette), au programme d'ajustement structurel le plus austère jamais exigé d'un pays européen. Alexis Tsipras ne démissionna pas. Il remporta de nouvelles élections en septembre, juste avant la mise en œuvre des premières mesures d'austérité du troisième programme. Depuis, il applique les prescriptions de ses créditeurs - les véritables gouverneurs de la Grèce -, creusant le désespoir d'une société atteinte dans sa substance, désorganisée et vaincue par ceux-là mêmes qui promettaient de la défendre.

Cet article analyse les principales mesures appliquées au système national de santé grec, secteur d'une importance capitale en période de crise des finances publiques et miroir dans lequel se reflètent les conditions d'existence des populations. Le texte questionne la rationalité en vertu de laquelle les gouverneurs de l'UEM persistent à « faire comme si » la dévaluation interne était la seule voie possible et à se montrer aveugles aux effets délétères de celle-ci. L'hypothèse, défendue ailleurs plus en détail (Burgi, 2014a), est que nous assistons à la culmination d'un effort long de désinstitutionalisation des régimes de protection sociale, poursuivi graduellement depuis au moins trois décennies, qui s'accélère à la faveur des choix politiques arrêtés par les puissances hégémoniques d'Europe pour gérer les effets de la crise financière de 2007-2008. Cet effort est lié à un projet néolibéral de refondation complète des principes, des modalités et des finalités de l'intervention de l'État d'après 1945. Également appelé « fondamentalisme de marché ${ }^{7}$ », il préconise la sujétion de toute la vie sociale et de toute la sphère publique, y compris l'État, aux mécanismes du marché. Parmi les nouvelles attributions de l'État, l'une des règles essentielles commande que sa politique sociale soit entièrement remodelée pour accompagner « de façon active » et porter à leur paroxysme les mécanismes de concurrence. Avant 2010, quasiment tous les gouvernements occidentaux, chacun à leur rythme et avec leurs modalités propres, ont cherché à pro-

7. Le fondamentalisme de marché s'enracine dans divers courants théoriques, dont l'ordolibéralisme, théorisé par des économistes allemands et autrichiens rejoints par Friedrich Hayek dès avant la seconde guerre mondiale. Longtemps marginales, leurs thèses ont trouvé leur point d'appui politique dans les « révolutions conservatrices » des années 1970 et 1980 impulsées par Thatcher et Reagan. 
gressivement reconfigurer leurs systèmes nationaux de protection sociale pour les conduire dans cette direction et les mener vers la constitution d'un nouveau « modèle social européen » dans lequel les prestations à vocation universelle des États sociaux construits après 1945 sont remplacées par un filet social minimal, par un « minimum vital » (Hayek, 1985 [1946]:89-90).

Ce minimum n'est pas conçu comme un moyen de lutte contre la précarisation et la paupérisation des masses car il n'est pas question de s'attaquer à leurs causes ni de revenir sur la régulation néolibérale. Au contraire, dans le cadre du «fondamentalisme de marché », le minimum vital a pour fonction de faire en sorte que personne, en principe, ne tombe définitivement hors-jeu - hors du jeu de la concurrence généralisée. Pour le dire autrement, le problème théorique et pratique posé à la gouvernementalité ${ }^{8}$ néolibérale dans la redéfinition des politiques de protection sociale est de savoir, non pas comment combattre le chômage de masse ou contenir l'extension des zones de précarité et de vulnérabilité, mais jusqu'où il est possible et/ou souhaitable d'abaisser le « seuil de pauvreté "absolue" " » en dessous duquel l'État devra imposer un filet social minimal, au sens d'un régime de soutien (et de contrôle étroit et punitif) des plus démunis, certes financé par la collectivité, mais juste suffisant pour le marché.

La déconstruction méthodique, quoique parfois anarchique, des institutions grecques de santé publique s'inscrit dans cette évolution. Au rythme dicté par les institutions de la troïka, les gouvernements grecs ont taillé dans les dépenses de santé « avec des couteaux de boucher», selon l'expression d'un ancien ministre de la Santé (2010-2012), Andreas Loverdos, et cela, au moment même où les déterminants sociaux de la santé - les conditions de vie, fortement dégradées sous l'effet des politiques austéritaires dans leur ensemble - se répercutaient sur la santé de la population. Pour reconfigurer le secteur, des recettes "clé en main » de la Banque mondiale et du FMI (partage des coûts, principe de dissociation entre acheteurs et fournisseurs, tarification à l'activité, privatisation des services...) ont été plaquées sur le système public de santé grec dans l'intention prioritaire de réduire les coûts, d'extraire des ressources et de réorienter les comportements vers la consommation d'assurances et de services privés.

8. La notion foucaldienne de « gouvernementalité » désigne, au-delà des structures politiques et de la gestion des États, et des formes instituées et légitimes d'assujettissement politique ou économique, un art de gouverner consistant à " conduire des conduites », à agir sur les possibilités d'action des individus et des groupes, et donc à structurer leur champ d'action éventuel.

9. L'expression est proposée par Michel Foucault (2004:207-213) dans un passage où il discute la question du minimum vital, qu'il appelle " clause de sauvegarde » ou de " non-exclusion ». La notion de pauvreté " absolue » ne s'entend évidemment pas au sens de la théorie économique. L'idée doit se comprendre comme une opération consistant à agir sur la paupérisation des masses, considérée comme un des effets de la régulation néolibérale, sans toutefois chercher à en modifier les causes. II s'ensuit que le tracé du seuil de pauvreté résulte d'un choix et d'une appréciation politiques. 
L'ampleur des coupes budgétaires et la logique qui leur est sous-jacente sont présentées dans un premier temps (I). Une deuxième partie étudie plus en détail les contradictions et les effets des politiques relatives aux médicaments et aux secteurs secondaire (hospitalier) et primaire (II). Abordant en dernier lieu la question des déterminants sociaux de la santé (III), la réflexion, centrée sur la mise en place d'un filet social équitable qui reste très largement insuffisant, débouche sur un questionnement portant sur le sens et les perspectives d'une société dite résiliente.

\section{Des « couteaux de boucher » pour tailler dans les dépenses}

Depuis sept ans, le secteur de la santé publique est l'une des principales cibles des programmes dits d'ajustement structurel dictés à la Grèce par les institutions de la troïka dans le cadre des mémorandums de 2010, 2012 et 2015. Le premier avait exigé que les budgets de santé publique passent de 6,8 \% du PIB en 2010 à 6,0 \% en 2012. À l'époque, des chercheurs internationalement reconnus avaient jugé « arbitraire » et «anormalement bas » un tel objectif (Stuckler, Basu, 2013, trad. fr. 2014 ; Karanikolos et al., 2013; Kondilis et al., 2012, 2013 ; Kentikelenis et al., 2014). Il fut cependant atteint en 2012 puis largement dépassé. En 2014, le ratio des dépenses de santé publique rapportées au PIB était de 4,9\%, son niveau le plus bas depuis 2004 . Il est remonté à 5,0\% en 2015, 5,1\% en 2016 et les prévisions pour 2017 le situent à 5,4\% du PIB. Cela se compare à une moyenne de 6,5\% dans l'Union européenne (UE) et à des ratios (stables) bien plus élevés dans les pays les plus riches de l'Union, notamment la France et l'Allemagne (tableau 1). On soulignera que la contraction des budgets de santé publique a été plus implacable encore en Grèce que ne l'indiquent ces pourcentages : dans la mesure où le PIB a lui-même perdu 27 points de pourcentage depuis 2010. En valeur réelle, les dépenses de santé ont donc chuté de près de moitié en quelques années.

Tableau 1. Part des dépenses publiques de santé dans le PIB (2009-2015) En \%

\begin{tabular}{|l|c|c|c|c|c|c|}
\hline Années & Grèce & Portugal & Espagne & Italie & France & Allemagne \\
\hline 2009 & 6,8 & 6,9 & 6,8 & 7,0 & 8,5 & 9,3 \\
\hline 2010 & 6,8 & 6,9 & 6,7 & 7,0 & 8,4 & 9,2 \\
\hline 2011 & 6,4 & 6,5 & 6,7 & 6,8 & 8,4 & 9,0 \\
\hline 2012 & 6,0 & 6,1 & 6,5 & 6,8 & 8,5 & 9,0 \\
\hline 2013 & 5,6 & 6,1 & 6,4 & 6,8 & 8,6 & 9,2 \\
\hline 2014 & 4,9 & 6,0 & 6,3 & 6,9 & 8,7 & 9,3 \\
\hline 2015 & 5,0 & 5,8 & 6,3 & 6,8 & 8,6 & 9,4 \\
\hline
\end{tabular}

Source : OCDE, https://data.oecd.org/healthres/health-spending.htm\#indicator-chart. 
Cette compression a donné lieu à une recomposition des dépenses de santé. Selon Giannis Kyriopoulos ${ }^{10}$, ancien doyen de l'École nationale de santé d'Athènes, alors que le financement des hôpitaux publics a chuté de plus de moitié entre 2009 et 2014 et que les dépenses totales de santé (publiques et privées) sont en forte baisse, le secteur hospitalier a connu un accroissement de sa part (+41\% entre 2008 et 2013) dans les dépenses totales, devenant (sans moyens supplémentaires) le dernier recours pour les malades. Cela reflète un moindre accès non seulement aux soins hospitaliers privés (qui ont baissé de $28 \%$ pendant la même période), mais encore aux soins primaires (services médicaux de base, soins dentaires, diagnostics, physiothérapies et autres) pour lesquels les dépenses ont chuté de $56 \%$ au cours de ces années. Dans le même temps, la désorganisation des structures de soin a accentué la corruption et la quête de passe-droits avec des paiements formels et informels aux médecins du secteur privé, en hausse de $52 \%$.

Aussi indispensables soient-elles, les données agrégées ne permettent cependant pas de saisir l'ampleur de la crise sanitaire. On constate par exemple en 2014 que la chute des dépenses publiques de santé après 2010 les ramènent, en valeur relative, à leur niveau de 2004 (graphique 1). Or en 2004, les Grecs, dans l'ensemble, avaient accès aux soins médicaux. Ce n'est plus le cas en 2014, et moins encore en 2017.

\section{Graphique 1. Part des dépenses publiques de santé dans le PIB en Grèce (1996-2014)}

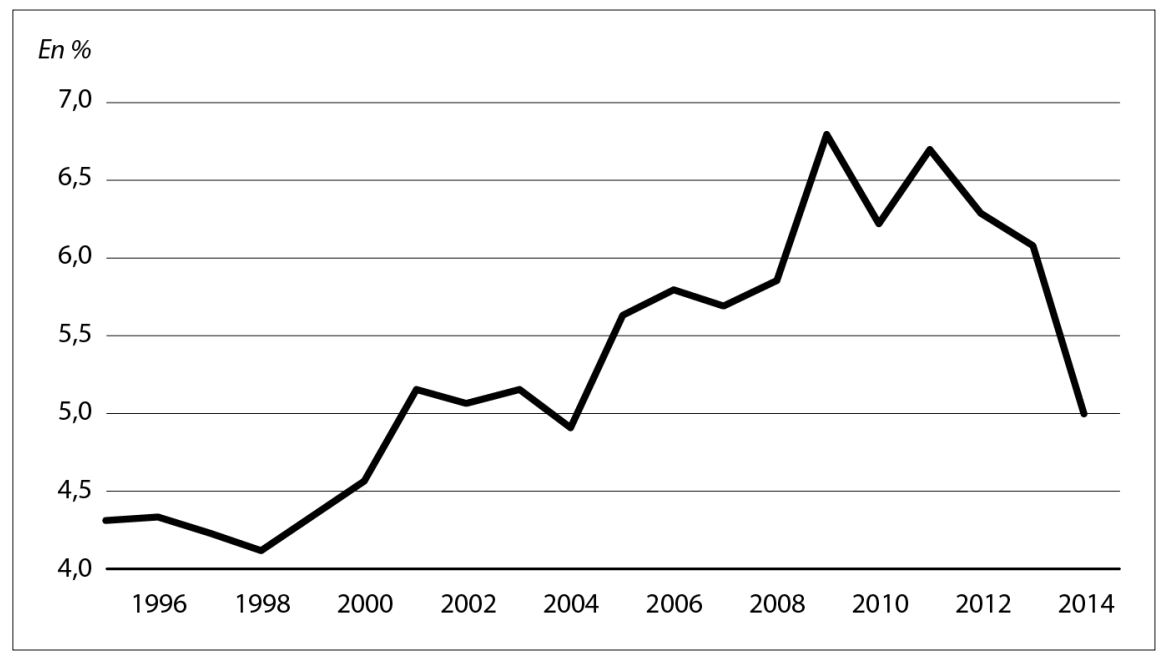

Source : Banque mondiale, http://data.worldbank.org/indicator/SH.XPD.PUBL.ZS?locations=GR.

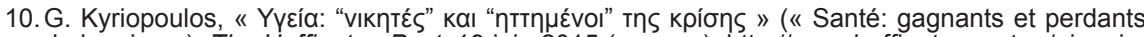
de la crise »), The Huffington Post, 19 juin 2015 (en grec), http://www.huffingtonpost.gr/gianniskyriopoulos/-_583_b_7619170.html. 
La différence entre 2004 et 2017 tient aux dimensions qualitatives du démantèlement récent des structures de santé publique - non-recours aux soins en temps utile, carences et dysfonctionnements des services médicaux... -, dont un des indices clés se constate dans l'augmentation significative après 2010 des cas de mortalité dus à des événements indésirables survenus en cours de traitement (Laliotis et al., 2016). Mais la santé publique dépend aussi très largement de facteurs sociaux plus généraux : accès à l'éducation, conditions de travail et loisirs, logement, perspectives d'avenir, état des communautés, des villages et des villes. Ces conditions structurelles de la vie quotidienne constituent « les déterminants sociaux de la santé et sont responsables pour une part importante des inégalités de santé entre pays et à l'intérieur des pays » (Commission on Social Determinants of Health [CSDH], 2008:1 ; Daniels et al., 1999). Or, dans le contexte des politiques austéritaires généralisées exigées par l'UE et ses membres les plus influents pour gérer les effets de la crise financière de 2008, la Grèce, classée première par l'OCDE (2015:126) pour sa "réactivité globale aux priorités de réformes » entre 2007 et 2014, est allée plus loin que tous les autres pays de l'organisation internationale dans la mise en place de mesures d'austérité, exceptionnellement sévères en ce qui la concerne. Au-delà des services de santé stricto sensu, elles ont provoqué une abrupte dégradation des conditions de vie (des déterminants sociaux de la santé) (comparaison des données Elstat ${ }^{11}$ entre 2012 et 2017 ; Burgi, 2014c). Pratiquement du jour au lendemain, le taux de chômage a grimpé pour devenir le plus élevé d'Europe (il frappe le quart de la population, la moitié des jeunes et sa durée s'allonge : les chômeurs étaient à 73,5\% de longue durée en 2015), les niveaux de vie se sont effondrés de plus de $30 \%$ en moyenne, la pauvreté relative et surtout l'extrême pauvreté ont connu une progression spectaculaire ${ }^{12}$, les services publics ont été démantelés et le droit du travail et de la négociation collective quasiment liquidé (Kapsalis, Kouzis, 2014), le tout entraînant une importante fuite des cerveaux (Koniordos, 2017) et des capitaux et une " catastrophe » sanitaire (expression de Médecins du Monde, notamment).

Les conséquences sur la santé des politiques d'ajustement structurel ont été largement ignorées, voire niées par les gouverneurs ${ }^{13}$ européens et grecs. Ils ont presque ${ }^{14}$ toujours soutenu, contre l'évidence, que les politiques austéritaires ne touchent en rien les services essentiels : " nécessaires » au

11. http://www.statistics.gr/en/living-conditions-in-greece.

12. Selon l'étude détaillée de Matsaganis et al. (2016), le taux de pauvreté extrême n'excédait pas $2,2 \%$ en 2009 ; il était de $8 \%$ en 2011 et de $15 \%$ en 2015 . On en trouvera une synthèse en anglais dans Georgakopoulos (2016). Sur l'évolution du seuil de pauvreté, voir Parlement hellénique (2014) et Matsaganis, Leventi (2013).

13. J'emploie le mot gouverneurs pour marquer le déploiement en un ordre de bataille quasi-militaire des injonctions et des mesures d'austérité.

14. Depuis septembre 2015, le gouvernement d'Alexis Tsipras tient un double discours, " réaliste » ou « résistant », selon l'auditoire auquel il s'adresse. 
bien commun, elles auraient au contraire permis de préserver l'avenir grâce à des gains d'efficience et d'efficacité du système de soins.

Cette ligne d'argumentation n'est pas nouvelle ni limitée à un pays. Elle reflète un changement profond dans la manière de concevoir et d'aborder les problématiques relatives à la santé publique. L'idéal inscrit dans la déclaration d'Alma Alta (1978) en vertu duquel la santé, considérée comme un droit humain fondamental, devrait être accessible à tous en fonction des besoins de chacun, s'est mué en une représentation de la santé assimilée à une transaction économique. Cette approche, portée par des institutions puissantes comme la Banque mondiale et le FMI, est devenue hégémonique dans les années 1990 et 2000. La première a réussi à imposer au monde sa vision économiciste de la santé et ces mêmes préceptes se retrouvent dans les prescriptions standardisées des programmes d'ajustement structurel du FMI : maximisation des prestations privées, frais modérateurs ${ }^{15}$, priorité aux marchés et à la concurrence. Le but serait d'accroître la rentabilité des dépenses afin de créer les conditions d'un développement économique soutenable. Cependant, comme le montre la littérature académique qui a analysé les conséquences de ces mesures, les dispositifs de type marchand ont accru plutôt que réduit les coûts, notamment les coûts bureaucratiques; ils ont miné la recherche médicale et les services publics de santé existants et approfondi les inégalités (Lister, 2008 ; Sachs, 2005 ; Commission on Social Determinants of Health [CSDH], 2008).

\section{Construction et déconstruction du système de santé}

Les reproches adressés aux opérations « clé en main » de restructuration des institutions de santé publique n'induisent pas qu'il ne faudrait pas améliorer ou réformer les systèmes existants. Ils portent sur la méthode, les finalités et les effets des mesures introduites sans considération pour les droits fondamentaux et le bien-être physique, mental et social des citoyens dans leur ensemble. S'agissant du système national de santé grec, il n’a jamais été particulièrement cohérent ou efficient. Mais les dispositions prises depuis 2010 par les gouvernements successifs sous l'égide de la troïka - politique du médicament, restructuration hospitalière, rationalisation des soins primaires - ont considérablement aggravé les problèmes de fonctionnement, d'efficacité et d'accès aux soins médicaux observables à la veille des mémorandums.

15. Les frais modérateurs désignent la partie des coûts assumée par les patients pour les services couverts par les régimes publics d'assurance maladie : ticket modérateur (représentant un pourcentage fixe du coût du service), copaiement (montant forfaitaire exigible quel que soit le coût du service, par exemple 5 euros), franchise (coût total assumé à hauteur d'un certain plafond), surfacturation, etc. 


\section{II.1. Déboires et succès du système national de santé grec (ESY) à la veille des mémorandums}

Créé en 1983, l'ESY représente incontestablement le plus important effort tenté en Grèce pour établir un véritable système national de santé. À l'origine, le projet ambitionnait d'unifier une pléthore de caisses professionnelles et de remplacer l'incohérente infrastructure de soins primaires existants par un réseau entièrement nouveau de centres de santé urbains et ruraux qui donneraient à tous les citoyens un égal accès aux soins, gratuits au point d'utilisation. Cependant, la résistance de groupes d'intérêt puissants (médecins pratiquant dans des cabinets privés, fonds d'assurance autonomes, fonctionnaires, syndicats, ainsi que des politiciens au pouvoir ou dans l'opposition) contraria cette visée initiale (Mossialos et al., 2005) et le système finalement mis en place associa de façon complexe trois types de structures : (a) des structures de type beveridgiennes financées par l'impôt (l'ESY proprement dit) ; (b) des organismes de type bismarckien regroupés dans le réseau des assurances sociales obligatoires financées par des cotisations de sécurité sociale; et (c) les services de santé privés ${ }^{16}$.

Avant 2010, l'ESY comprenait : 201 centres de santé ruraux et trois centres de santé urbains qui formaient des unités décentralisées des hôpitaux régionaux de l'ESY ; 1478 postes médicaux ou chirurgicaux rattachés aux centres de santé ; et les cliniques ambulatoires de 140 hôpitaux publics. Les centres de santé, les postes médicaux et chirurgicaux offraient à la population rurale des services préventifs, curatifs, d'urgence et de réhabilitation gratuits au point d'utilisation. Les cliniques ambulatoires des hôpitaux publics proposaient des services de spécialistes ou de diagnostic à la population urbaine et semi-urbaine. De jour, l'accès était gratuit ou soumis à une participation financière minimale et, de nuit, il fallait acquitter un copaiement.

Le réseau de sécurité sociale consistait en 36 caisses professionnelles couvrant les soins primaires de $95 \%$ de la population selon diverses formules. L'affiliation à ces caisses, structurées par branche ou par catégorie socio-professionnelle, était obligatoire. Par ordre d'importance, la première des quatre principales caisses était l'IKA (ou Fondation de Sécurité sociale, créée en 1934), la caisse la plus importante des travailleurs du secteur privé. Elle avait sa propre infrastructure de soins et ses propres médecins (surtout des spécialistes), tous salariés et autorisés à ouvrir des cabinets privés à mi-temps. Les trois autres caisses couvraient respectivement les travailleurs agricoles (l'OGA), les professions libérales (l'OAEE) et les employés du secteur public (l'OPAD). Tous les centres de santé achetaient partiellement ou exclusivement des services auprès de laboratoires ou de médecins

16. La description qui suit est empruntée pour l'essentiel à Kondilis et al. (2012). 
privés. Les assurés avaient gratuitement accès à une vaste gamme de services, principalement curatifs et de diagnostic. Si les patients étaient redirigés vers des laboratoires et médecins privés, ils versaient un copaiement.

Enfin, le secteur privé comprenait environ 25000 médecins, 12000 dentistes, entre 400 et 700 laboratoires et 167 hôpitaux avec leurs départements ambulatoires. Des centres de diagnostic privés hautement rentables contrôlaient presque tout l'équipement biomédical du pays. Ces centres et les médecins privés passaient des contrats avec les caisses d'assurances sociales et les assurances privées et facturaient leurs interventions sur la base d'un forfait fixe par service payé conjointement par les usagers et les caisses. Le secteur primaire privé absorbait plus de $65 \%$ des dépenses privées totales de santé (Kondilis et al., 2012).

Pour diverses raisons, ce système compliqué, fragmenté et peu coordonné connaissait des difficultés permanentes avant 2010. La part importante du secteur privé, la carence de généralistes, d'importantes différences dans le nombre et la qualité des services et dans l'étendue de la couverture garantis par les différents régimes d'assurance, et de réelles carences dans les zones rurales rendaient le système inefficient et inégalitaire. En outre, les très faibles salaires du personnel de l'ESY et du réseau primaire des assurances sociales avaient causé des problèmes structurels : difficultés permanentes de recrutement dans les hôpitaux, sous-effectifs importants, surtout d'infirmiers et de docteurs, manque d'unités de soins intensifs (du fait des sous-effectifs), longues listes d'attente... conduisant à l'habitude de glisser une enveloppe (fakelaki) entre les mains des médecins afin de contourner la liste d'attente et (espérer) obtenir un meilleur traitement.

S'il ne fait pas de doute que le système national de santé avait besoin de changement, il avait malgré tout contribué à une amélioration remarquable de la santé publique entre 1983 et 2009. Les données de l'Organisation mondiale de la santé (OMS) indiquent une importante progression de l'espérance de vie pendant cette période grâce à la chute des mortalités évitables (causées par des maladies traitables), en particulier un remarquable déclin de la mortalité infantile et néonatale (passées respectivement de 17,94 à 2,65 et de 13,58 à 1,79 morts pour 1000 naissances), et de la mortalité postnatale et maternelle. De même, dans le Rapport sur la santé dans le monde publié par l'OMS en 2000, la Grèce figurait en excellente position dans le classement des 191 pays membres de l'Organisation selon la qualité des soins médicaux dispensés. Elle était alors $14^{ }$. La France figurait au premier rang, suivie par l'Italie ; l'Espagne était septième ; le Portugal, précédé de la Norvège, $12^{\mathrm{e}}$. La Suède arrivait en $23^{\mathrm{e}}$ position, l'Allemagne en $25^{\mathrm{e}}$, les États-Unis, pays notoirement connu pour son système de santé mercantile, $37^{\mathrm{e}}$. 


\section{II.2. Restrictions budgétaires et délais de paiement : des médicaments de moins en moins accessibles}

Les dysfonctionnements de l'ESY avaient favorisé une surconsommation de médicaments en Grèce. En 2009, les dépenses médicamenteuses étaient les plus élevées des pays de l'OCDE (2,4 \% du PIB contre 1,6\% en moyenne). Ce phénomène s'explique notamment par les pratiques des médecins du réseau primaire des assurances sociales : ils avaient été nombreux à chercher une compensation à leurs faibles salaires ${ }^{17}$ en ouvrant les après-midis des cabinets privés. Du coup, les consultations publiques du matin devenaient un vivier de recrutement de patients pour leurs consultations privées : schématiquement, le patient était rapidement reçu le matin avant de repartir avec un rendez-vous pour une consultation privée, ou à défaut avec une grosse ordonnance de médicaments.

La réduction des dépenses pharmaceutiques fut programmée dès le premier mémorandum. La troïka voulut une baisse de près de moitié des dépenses en deux ans (de 4,37 milliards d'euros en 2010 à 2,88 milliards d'euros en 2012 - cet objectif fut atteint) puis à 2 milliards d'euros en 2014 (Kentikelenis et al., 2014) en agissant sur les prix, les prescriptions et le monitoring (Carone et al., 2012:50-52). Parmi les autres mesures figurait une liste régulièrement modifiée de médicaments remboursables conçue pour servir l'objectif prioritaire, en Grèce comme ailleurs, du recours aux génériques; un système de rabais sur tous les médicaments vendus aux caisses de sécurité sociale ; et un droit de recouvrement par l'État lorsque les dépenses du budget pharmaceutique public excédaient un plafond périodiquement révisé.

Le prix des médicaments est maintenant basé sur la moyenne des trois plus bas prix de l'Union européenne. Des économies substantielles ont ainsi été réalisées sans toutefois assurer un meilleur accès du public aux médicaments. Les raisons en sont complexes. Les hôpitaux délabrés ne sont pas en mesure de commander des médicaments dont les patients ont besoin et laissent ces derniers essayer de résoudre le problème. Les pharmacies sont en difficulté : d'un côté, elles ont accumulé des dettes du fait des délais de remboursement des organismes de sécurité sociale (actuellement, quatre à cinq mois de retard en moyenne, mais cela peut atteindre dix ou onze mois) qui leur doivent un total évalué en 2015-2016 à 500 millions d'euros (Karamanoli, 2015 ; Mantas, entretien 2016). De l'autre côté, les fournisseurs accordent un délai de un à trois mois, mais exigent le plus souvent un règlement immédiat. Les pharmacies sont alors forcées de payer leurs commandes d'avance en attendant le remboursement des assureurs sociaux, ou

17. 1100 euros mensuels dans les unités de soins de l'IKA, sans augmentation jusqu'à la retraite, non comprise l'assurance maladie obligatoire entièrement à leur charge (contrairement à tous les autres salariés), et sans indemnités en cas de licenciement. 
de s'organiser informellement avec d'autres pharmacies, parmi lesquelles les pharmacies solidaires aujourd'hui très bien organisées ${ }^{18}$, pour trouver ou échanger les médicaments ; ou encore d'exiger des patients d'avancer les fonds; ou enfin de les envoyer tenter leur chance ailleurs. Ces solutions sont fragiles : un très grand nombre de pharmacies a fermé. Beaucoup d'autres ont survécu en apparence mais ont été rachetées par des firmes multinationales de grossistes privés. Ces derniers ont réagi aux bas prix et aux longs délais d'apurement des créances en se tournant vers d'autres marchés plus lucratifs. Pendant un temps, les fabricants ont fixé des quotas censés couvrir les besoins du marché grec, et, par conséquent, ils ne livraient pas nécessairement les commandes en totalité. Pour se justifier, ils disaient ne pas avoir suffisamment de stocks, et parmi eux quelques-uns avaient arrêté de vendre des médicaments coûteux à la Grèce. Un problème particulier se pose au sujet des nouveaux médicaments entrant sur le marché grec (83 ces trois dernières années) parce que la Grèce figure au plan international parmi les pays de référence pour l'établissement et la négociation de leur prix ${ }^{19}$. Ainsi, la firme Novartis s'est alliée avec des agents publics et des docteurs grecs pour vendre en Grèce certains nouveaux produits à des prix exorbitants qui lui ont assuré des profits élevés dans des pays plus peuplés (comme par exemple la Turquie, et plus encore le Brésil). Le scandale Novartis ${ }^{20}$ fait actuellement l'objet d'une enquête pour établir les faits et déterminer les responsabilités. En attendant, des initiatives internationales sont prises pour tenter de modérer les appétits des fabricants du fait de la crise générale des finances publiques. En Grèce, la pression (et le mécontentement des fabricants) est encore plus forte parce que, en sus de son droit de recouvrement, l'État demande aux fabricants une réduction de $25 \%$ pour chaque nouveau médicament dont le prix est fixé en Grèce.

Ajoutons enfin que l'industrie pharmaceutique grecque est structurellement vulnérable aux firmes multinationales et aux politiques gouvernementales. Elle produit des génériques de haute qualité et constitue un vivier de main-d'œuvre effectif et potentiel, mais elle n'est pas en mesure de se défendre contre des menaces telles que le dumping sur les prix des firmes pharmaceutiques mondiales. Elle a aussi été affaiblie par le droit de recouvrement de l'État dont le montant pour 2014 a été estimé à $30 \%$ du budget national pharmaceutique (Anastasaki et al., 2014). Des opportunités de croissance endogène se perdent ainsi, tandis que la société est dépossédée d'un fournisseur de qualité.

Pour aggraver la situation, les nouvelles politiques ont transféré une partie des coûts aux patients qui doivent assumer une part croissante de la

18. Elles sont brièvement évoquées à la fin de ce texte.

19. Sur ces pratiques, voir par exemple Gandjour (2013).

20. Voir par exemple http://www.cardiobrief.org/2016/03/29/us-doj-expands-investigation-into-phony-novartis-speaking-events/. 
dépense totale non remboursée au risque de se priver de soins. Le panier de soins a été modifié pour introduire des frais modérateurs et exclure certains produits et services, en particulier les tests cliniques et pharmacologiques, de la couverture publique (les assureurs privés ont aussi restreint leur couverture). En moyenne, le ticket modérateur acquitté par les assurés est passé de $9 \%$ du prix du médicament en 2011 à $25 \%$ en 2013 et 35 à $40 \%$ en 2015. Dans les cas extrêmes, le ticket modérateur peut atteindre $75 \%$ du prix du médicament. En raison des effets combinés des pénuries, des stratégies des firmes, et de la règle stricte de l'alignement du médicament sur les trois plus bas prix de l'UE, les médicaments prescrits sont régulièrement introuvables sur le marché. Par exemple, ce sont les vaccins, surtout les vaccins pour enfants (en 2017), ou encore l'insuline, les anticoagulants, les produits antidiabétiques et anticancéreux, les immunosuppresseurs et d'autres produits essentiels qui ne sont plus en circulation ou ne s'obtiennent que très difficilement. Le dysfonctionnement du système est particulièrement dangereux pour les personnes atteintes de maladies chroniques.

\section{II.3. Les hôpitaux en sursis permanent}

Des mesures drastiques ont été introduites pour restructurer les hôpitaux publics et le reste de l'ESY. Au cours des dernières années, on a assisté à la fermeture de grands hôpitaux à Athènes, Thessalonique et ailleurs, la suppression et/ou la fusion d'un grand nombre de cliniques ou d'unités spécialisées, le regroupement de centaines de laboratoires, et l'élimination d'au moins 2000 lits. Des mécanismes de surveillance managériale ont été mis en place : les budgets des hôpitaux sont maintenant gérés par une firme privée (Karakioulafis, 2014:90) et des techniques variées permettent d'inspecter l'activité hospitalière et celle des médecins, dont un système de collecte mensuelle des données pour contrôler l'activité et les dépenses hospitalières à travers des procédures électroniques obligatoires. Comme ailleurs en Europe (Math, dans ce numéro), deux instruments clés ont été actionnés par les gouverneurs pour ralentir la croissance des dépenses hospitalières : la tarification à l'acte et la compression du personnel.

\section{La tarification à l'activité}

La tarification à l'activité ou diagnosis related groups (DRGs) est un outil budgétaire importé des États-Unis au début des années 1980 qui s'est substitué au prix de journée habituellement pratiqué en Europe. Elle s'est imposée partout, bien que la recherche scientifique et les évaluations indépendantes mettent depuis longtemps en lumière sa nuisibilité tant pour les finances publiques que pour les patients. Elle consiste à lier directement les recettes des hôpitaux au volume d'activité (nombre d'actes et de 
consultations) enregistrées pour chaque groupe homogène de malades. Le critère est purement comptable et ne permet pas de distinguer entre une activité technique, facilement quantifiable, mesurable, et une autre plus complexe exigeant plus de temps et l'appel à des compétences pluridisciplinaires. En France, par exemple, où un financement analogue a été introduit, toute consultation, pour être rentable, ne devrait pas dépasser douze minutes. Dans le contexte de l'injonction politique d'un retour à « l'équilibre financier », il n'est donc plus vraiment question de soins mais d'augmentation du nombre d'actes rentables (Grimaldi, 2009). En Grèce aussi, cette politique organise le renoncement à la qualité de bien public inhérente aux services de soin pour les transformer en entreprises capitalistes (Ioakeimoglou, 2010).

Cette transformation est cependant coûteuse. Comme le note le neurologue Makis Mantas (entretien, 2014), coordinateur jusqu'en juillet 2015 du programme de soins primaires de Syriza, le système de paiement à l'acte «... a accru les déficits publics [grecs] et multiplié les coûts hospitaliers par sept. Il favorise les hôpitaux privés. Prenez le cas du strabisme. Il s'agit d'un acte très simple. Dans le passé, l'opération coûtait entre 70 et 90 euros en Grèce. Aucun hôpital privé ne s'y intéressait. Aujourd'hui, la même opération coûte dix fois plus cher. Soudainement, les hôpitaux publics qui prenaient en charge toutes ces opérations le font de moins en moins; ils cèdent la place aux hôpitaux privés. »

Les hôpitaux privés y trouvent leur compte et en tirent des profits élevés parce qu'ils peuvent facilement se spécialiser dans les traitements relativement simples et peu risqués de maladies courantes et prévisibles. Ce qui pénalise les hôpitaux publics et universitaires auxquels incombent les traitements plus complexes, coûteux et risqués, alors que leurs ressources et celles de la recherche médicale ont diminué.

\section{Des coupes drastiques dans les salaires et les effectifs du secteur public de santé}

Autre instrument majeur visant la baisse des coûts, les coupes salariales et la liquidation des droits des travailleurs dans tout le secteur public ont été, dès le premier mémorandum, l'une des principales priorités de la troïka. En 2011, il apparaissait déjà que la chute des dépenses publiques hospitalières avait été obtenue spécialement au moyen d'une diminution de $75 \%$ des coûts salariaux (plutôt que d'un accroissement de l'efficience) (Kondilis et al., 2013). Les salaires des professionnels de santé publique, qui étaient les plus faibles d'Europe occidentale avant la crise financière, ont été réduits d'au moins $40 \%$ depuis 2010. Charis Matsouka (entretien, $2014^{21}$ ) affirmait qu'elle gagnait 2000 euros par mois, frais professionnels

21. Directrice du service d'hématologie de l'hôpital universitaire général Alexandra à Athènes. 
inclus, alors qu'elle avait atteint le sommet de la carrière hospitalière. Aujourd'hui, le salaire moyen d'un nouveau médecin référent ou d'un professeur de médecine débutant s'élève environ à 1100 euros (Ifanti et al., 2014; entretiens, 2016, 2017).

La compression du personnel dans le secteur de la santé publique a été dramatique, avec une perte de $30 \%$ des effectifs consécutive au gel des embauches, au non-remplacement de fait des travailleurs partant à la retraite et au non-renouvellement des contrats temporaires. En 2011, l'Association médicale d'Athènes estimait qu'en cette seule année, 26000 agents du secteur de la santé publique, dont près de 9100 médecins, allaient perdre leur travail (Triantafyllou, Angeletopoulou, 2011). La réduction du nombre de médecins a été beaucoup plus importante que celle prévue par la troïka (Correia et al., 2015) à cause de la détérioration rapide de l'environnement et des conditions de travail. Nombre de docteurs et d'infirmiers ont pris des retraites anticipées. À cela s'ajoute un exode massif depuis 2010 de jeunes grecs hautement qualifiés, parmi lesquels des médecins spécialistes et autres personnels médicaux à la recherche de conditions de travail meilleures hors de Grèce. On estime à plus de 7500 le nombre de médecins grecs ayant émigré jusqu'en 2014, notamment vers l'Allemagne où ils sont employés dans des postes en deçà de leurs qualifications et à des taux de rémunération inférieurs à ceux de leurs collègues ${ }^{22}$.

\section{Des conditions de travail et sanitaires déplorables}

Les sous-effectifs, un afflux considérable de patients et les pénuries ont poussé les hôpitaux publics au point de rupture. Les conditions de travail y sont précaires et dangereuses. Les hôpitaux sont à court des fournitures les plus élémentaires : draps, ciseaux, antalgiques, tensiomètres, équipement stérilisé, médicaments vitaux, dépistage du cancer et équipement approprié pour les interventions chirurgicales... Le nombre d'heures travaillées a considérablement augmenté. Le temps de travail des médecins, incluant les périodes de garde, peut atteindre 32 heures ininterrompues, et lorsqu'ils sont en service d'astreinte la durée du travail peut atteindre 93 heures par semaine. Alertée, la Commission européenne a porté le problème devant la Cour de justice qui a jugé ces pratiques illégales ${ }^{23}$. Jusqu'ici, cela n’a pas été suivi d'effet : alors que taux de chômage est élevé parmi les médecins et le personnel soignant, les recrutements de médecins statutaires n'ont pas augmenté, des milliers d'embauches sont toujours en attente et les internes quittent l'ESY. À la mi-2017, une loi destinée à réduire le temps

22. Les données de l'OCDE sur l'émigration du personnel médical ne sont pas disponibles pour la Grèce.

23. Jugement de la Cour (Neuvième Chambre) du 23 décembre 2015, European Commission $v$ Hellenic Republic, case C-180/14. La directive 2003/88/CE dispose qu'une période de travail de 24 heures doit être entrecoupée d'au moins 11 heures consécutives de repos et que le travail ne doit pas excéder 48 heures par semaine. 
de travail légal des médecins grecs pour le rendre conforme aux normes européennes (48 heures) était en préparation. Mais comme il n'est toujours pas possible de recruter du personnel statutaire, le projet de loi prévoit une option en vertu de laquelle les médecins qui le souhaitent auront la possibilité de travailler 60 heures hebdomadaires à condition de signer un document attestant qu'ils font eux-mêmes le choix de travailler plus (auparavant, ils y étaient obligés). Par ailleurs, la pratique consistant à recruter du personnel non statutaire auto-employé sur des contrats à court terme se généralise. Des unités de soins intensifs, des laboratoires et même des départements hospitaliers entiers ferment ou menacent constamment de fermer. Les divers services luttent constamment pour obtenir les financements indispensables à leur survie. Comme le dit une femme médecin dans un entretien :

« Nous devons sans arrêt nous battre pour obtenir des fonds additionnels du gouvernement. (...) Ça se passe tous les trois mois. Donc nous avons constamment un horizon de trois mois devant nous. C'est épuisant. Et déprimant...»

Certains centres publics de traitement du cancer n'arrivent même pas à nourrir leurs patients. Le manque de ressources les amène parfois à fermer abruptement leurs services à divers moments de la journée et à annuler des consultations sans les reporter à une autre date. En janvier 2016, l'hôpital universitaire général Laiko à Athènes a renvoyé des dizaines de patients atteints du cancer parce qu'il ne pouvait pas pratiquer les chimiothérapies vitales prévues pour eux ${ }^{24}$. Dans certaines circonstances extrêmes, des nouveau-nés ont été retirés à leur mère jusqu'à ce qu'elle puisse payer la facture hospitalière ; le cardiologue Georgos Vichas, qui dirige la clinique métropolitaine solidaire d'Hellinikon, a rapporté des situations où des patients atteints du cancer avaient été expulsés de l'établissement de soins où ils devaient subir une opération chirurgicale parce qu'ils ne pouvaient pas payer 1800 euros. Conséquence de la forte proportion de personnes non assurées, ces dernières situations sont cependant restées exceptionnelles grâce à l'ingéniosité des médecins grecs qui trouvent des moyens créatifs de contourner les régulations.

\section{II.4. Le démantèlement du système de soins primaires}

Inégalitaire et fragmenté avant 2010, le réseau des services de soins primaires a connu, depuis, une série de réorganisations. En 2011-2012, les quatre principales caisses d'assurances sociales (IKA, OGA, OAEE et OPAD) furent transférées avec leurs effectifs et leur infrastructure à une nouvelle et unique Organisation nationale pour la prestation de services

24. « Yet Again, Cancer Patients in Desperation at Laiko Hospital », MCCH, April 11, 2016, http:// www.mkiellinikou.org/en/2016/04/11/yet-again-cancer-patients-in-desperation-at-laiko-hospital/. 
de santé (EOPYY). L'intégration des structures de soins primaires avait été espérée depuis au moins la création de l'ESY en 1983 car elle portait une promesse d'universalité et d'égalité d'accès à la santé. La promesse ne fut pas honorée dans le contexte des mémorandums. Légalement obligées de déposer $77 \%$ de leurs avoirs disponibles à la Banque de Grèce, les caisses d'assurance maladie absorbées par l'EOPYY perdirent 53,5\% de leurs avoirs au moment de la restructuration (" la décote ») d'une partie de la dette grecque en mars 2012. Elles ne reçurent aucune compensation pour leurs pertes (environ 10 milliards d'euros en trois mois), contrairement aux banques. Par ailleurs, la diminution des prestations couvertes par les caisses, l'augmentation des frais modérateurs, la compression des personnels soignants et les baisses de salaire détériorèrent, comme dans le secteur hospitalier, la qualité des prestations et l'accès aux soins primaires publics (Kaitelidou, Kouli, 2012 ; Kondilis et al., 2013).

En 2014, dans la ligne des principes promus par la Banque mondiale, le ministre de la Santé, Adonis Georgiadis, dissocia les fonctions d'acheteur et de fournisseur ou prestataire de soins. La fonction d'acheteur resta de la compétence de l'EOPYY, tandis que les prestations de santé furent confiées à un nouveau Réseau national de soins de santé primaire (PEDY). Le ministre manœuvra habilement pour provoquer le départ « volontaire » de la moitié des médecins des centres de santé intégrés à l'EOPYY deux ans plus tôt. En février 2014, il ferma du jour au lendemain toutes les unités du réseau de soins primaires et renvoya chez eux quelque 6500 à 8000 médecins. Il annonça que ceux-ci pourraient être embauchés à l'ouverture du nouveau réseau (PEDY), à condition d'accepter des contrats de travail à plein temps et de fermer leurs cabinets privés. L'idée de créer un statut d'emploi exclusivement public dans le secteur des soins primaires était populaire et en général bienvenue, mais cela impliquait que les docteurs dorénavant se contentent du même (bas) salaire (1 100 euros), renoncent à des sources supplémentaires de revenus (en fermant leurs cabinets) et oublient les perspectives de carrière ${ }^{25}$. Ils furent nombreux à " s'autolicencier », pour citer la formule d'un de mes interlocuteurs, c'est-à-dire à « choisir » de perdre leur emploi. Aujourd'hui, le réseau PEDY compte quelque 2700 médecins.

En dehors de réussir un « coup » avec la suppression d'un nombre conséquent d'employés du secteur public, l'initiative d'Adonis Georgiadis s'est révélée problématique. D’abord, les structures de soins primaires

25. Pour cette raison, les médecins et le personnel soignant avaient depuis novembre 2013 lancé des grèves reconductibles. Ils protestaient contre les plans du ministre et les licenciements mises en disponibilité et mobilités forcées qui se profilaient. Nombre d'entre eux avaient cependant gardé ouverts leurs cabinets privés, ce qui a peut-être délégitimé le mouvement aux yeux d'une partie de l'opinion publique, les docteurs pouvant donner l'impression d'être mus par des motivations individuelles dans ce conflit. 
(c'est-à-dire principalement celles de l'ancienne IKA) ont pratiquement cessé de fonctionner en raison de l'importance des sous-effectifs. Autrefois dotées de moyens, notamment humains, suffisants, elles sont désormais pratiquement vides. Par conséquent, de très nombreux patients doivent attendre longtemps pour une très brève consultation ${ }^{26}$. Les médecins n’ont pas le temps de faire davantage que de contrôler les carnets de santé ou les anciennes ordonnances, et en faire de nouvelles. De plus, ils sont étroitement surveillés par une plateforme électronique : ils ne peuvent pas prescrire de médicaments ou d'examens au-delà de ce qu'autorise leur budget ${ }^{27}$. S'ils s'y hasardent, le système commence par lancer un avertissement avant de se bloquer, et le médecin, alors passible d'une amende, ne peut plus rien prescrire. Dans les services de santé primaire (comme à l'hôpital), des auxiliaires précaires sont embauchés pour des périodes courtes allant de huit mois (ce qui évite de les garder l'été) à un an et demi au mieux : ceux-là ne veulent pas risquer leur emploi ou avoir une amende. Les autres ${ }^{28}$ sont également piégés par le système, de sorte que la plupart des médecins ne peuvent pas soigner leurs patients. Si nécessaire, ils les envoient à l'hôpital où il est encore possible de prescrire librement des médicaments ou des examens. Mais c'est un cercle vicieux, car les médecins hospitaliers estiment à juste titre qu'ils n'ont pas le temps de recevoir les gens pour simplement leur délivrer des ordonnances, et que ce n'est pas leur rôle.

Même quand ils n'étaient pas dirigés à l'hôpital par leur médecin traitant, et parce que les soins primaires ne fonctionnent pas, les patients se sont massivement tournés vers les urgences hospitalières. C'est le deuxième grand écueil de la réorganisation conduite par Adonis Georgiadis. Pour décongestionner les urgences et pallier les conséquences des souseffectifs dans les soins primaires, le ministre de la Santé a cru trouver une solution en incitant les médecins du secteur privé à passer des conventions avec l'EOPYY : ils s'engageaient à examiner 200 patients par mois contre une rémunération de 2000 euros mensuels. Cette mesure extrêmement coûteuse pour le système de santé publique s'est aussi avérée très inefficace car la plupart des médecins «se débarrassaient » des 200 patients en une semaine, sinon plus vite, et pendant le restant du mois les malades n'avaient

26. Il faut se représenter ce que cela signifie : j'ai vu un médecin généraliste s'occuper de 24 personnes en une heure un quart, ce qui revient à une durée moyenne de trois minutes par consultation. Ce n'est pas exceptionnel. Le travail du médecin est aussi pénible que l'est la situation pour le malade. Les patients pour la plupart savent ce qu'ils ont et viennent seulement renouveler leurs ordonnances. Mais il y a toujours au moins une minorité qui ne sait pas ce qui lui arrive et qui ne trouvera ni l'attention ni l'écoute dont elle a besoin.

27. Il est alloué à chacun en fonction de sa spécialité.

28. Ceux qui ont accepté les conditions posées par Adonis Georgiadis et ont été embauchés dans le nouveau réseau national (PEDY) après avoir le cas échéant fermé leur cabinet privé, et un second (petit) groupe de médecins et soignants qui avaient réintégré les services de soins primaires sur la base de plusieurs jugements des tribunaux condamnant le ministre pour leur licenciement de fait. Ces derniers ont bénéficié des mêmes conditions de travail (salaire et possibilité de conserver leur cabinet privé) pendant plusieurs années éventuellement renouvelables. Les personnels soignants ont donc des statuts d'emploi différenciés et inégalitaires. 
d'autre choix que de payer le prix d'une consultation privée, d'aller à l'hôpital ou de tenter leur chance dans un centre de soins primaires.

Telle était encore la situation au printemps 2017 quand l'actuel ministre de la Santé, Andreas Xanthos, lançait un nouveau projet de loi quadriennal dit «Soins primaires de santé » ${ }^{29}$. Son objectif prioritaire consiste toujours à désengorger les urgences hospitalières mais le gouvernement entend aussi remettre à plat tout le système en vue de son amélioration (encadré). L'initiative est bienvenue : elle simplifie l'organisation des soins primaires, vise leur intégration à un système national de santé unique et pourrait corriger certains dysfonctionnements. Mais les fortes contraintes budgétaires indiquent que la perspective reste celle d'un rationnement des soins de santé publique. Les besoins du pays tout entier ne sauraient être couverts par le recrutement de seulement 1300 nouveaux médecins, même si les profils privilégiés (généralistes, internistes, pédiatres) correspondent à un manque avéré, tant le déséquilibre est grand entre, d'un côté, les spécialistes en surnombre et, de l'autre, les généralistes souvent inexistants dans les services de soins primaires. Avec seulement trois praticiens, le rôle des unités locales de santé risque de se ramener à celui de pourvoyeur d'ordonnances et de garde-barrière empêchant le tout-venant de se rendre spontanément aux urgences hospitalières. On ne sait pas encore ce que devrait impliquer la restructuration de ces dernières, actuellement à l'étude. Elles pourraient devenir des unités autonomes dotées d'un personnel propre, distinct des équipes soignantes des hôpitaux. Dans l'ensemble, la précarisation des personnels soignants ira en augmentant. Même si les contrats des médecins conventionnés sont révisés, il manquera au système national de santé, et singulièrement au secteur des soins primaires, la masse critique indispensable à des prestations publiques de qualité. La dépendance à l'égard du secteur privé n'en sera que renforcée. On s'achemine très probablement vers un système à deux vitesses comprenant des services publics rudimentaires, sinon gratuits du moins à faible coût, et des prestations privées ou mixtes accessibles seulement aux plus aisés.

De fait, la logique d'une participation maximale du secteur privé s'installe progressivement dans le secteur des soins primaires. Les dépenses non remboursables se multiplient. Les copaiements pour les consultations ambulatoires sont passées de 3 à 5 euros en 2011 (le gouvernement Syriza a supprimé cette taxe qui vient d'être réintroduite). Les prescriptions médicales sont limitées à trois médicaments. S'il en faut davantage, le médecin doit établir une ou plusieurs nouvelles ordonnances, chacune coûtant 1 euro au patient. Il y a aussi d'autres coûts moins visibles, comme le prix

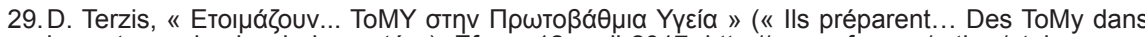
le secteur primaire de la santé »), Efsyn, 12 avril 2017, http://www.efsyn.gr/arthro/etoimazoyntomy-stin-protovathmia-ygeia. Les ToMY sont des « unités locales de santé » (voir encadré). 


\section{Encadré}

\section{Le programme quadriennal « Soins primaires de santé »}

Il ne devrait y avoir désormais que deux niveaux d'accès aux soins primaires. À la base, des " unités locales de santé " (ToMY) mises en place dans tout le pays et installées dans les centres existants (IKA) ou dans de nouvelles structures seront encadrées par au moins deux, sinon trois médecins, prioritairement un·e généraliste, un·e pathologiste et un·e pédiatre. Au total, 239 unités locales devraient être créées d'ici trois ans, dont 50 à 60 d'ici la fin 2017. Là où de telles unités n'existeraient pas, des médecins privés contractants de l'EOPYY devront combler les besoins selon des modalités qui restent à définir. Le ministre estime que les unités locales couvriront $30 \%$ des soins, les médecins privés les $70 \%$ restants, et il se donne pour but d'inverser ces proportions dans quatre ans. Les unités locales pourraient selon lui diminuer d'environ cinq millions et demi le nombre de consultations dans les services hospitaliers.

À un deuxième niveau, des " centres de santé " plus conséquents et comprenant plus de spécialités que dans les unités locales se substitueront aux services existants dans les provinces et les zones rurales, ainsi qu'aux anciennes structures du réseau national de soins primaires - c'est-à-dire le PEDY, dont la dénomination disparaît (elle n'est jamais passée dans le langage courant qui se réfère tantôt à l'EOPYY et tantôt à l'IKA pour désigner les centres des soins). Ils seront ouverts 24 heures sur 24. Le gouvernement espère avoir créé 240 centres de santé dans tout le pays, y compris à Athènes, d'ici la fin de l'année 2017.

Le coût total de l'opération est estimé à 300 milliards d'euros. Elle devrait bénéficier de crédits garantis par l'UE auxquels s'ajoute l'apport, en augmentation progressive, du budget de l'État. Cela inclut le recrutement de 3000 personnes, dont 1300 médecins. Les postes mis au concours ciblent des généralistes, des internes et des pédiatres. Ils seront payés sur la base du statut ESY d'assistant de catégorie A à l'hôpital, soit 1500 à 1600 euros net par mois.

d'un appel téléphonique pour programmer un rendez-vous chez le docteur. Il sera bientôt possible de le faire par voie électronique, solution difficile pour les personnes âgées ou démunies. L'assurance privée croît lentement dans le marché des soins primaires avec des formules bon marché. Par exemple, on a vu des journaux comme Proto Thema ou Anexartisia offrir aux lecteurs dans leurs éditions du week-end des coupons à collectionner pour obtenir une carte de santé " gratuite » donnant un accès annuel limité et à faible coût à des médecins et des centres privés de diagnostic. Les banques aussi offrent à leurs clients différents types de polices d'assurance maladie (selon un éventail de prix variant de 85 à 800 euros annuels). 
Les montants de ces contributions acquittées par le patient peuvent paraître négligeables. Cependant, elles pourraient bien avoir un but pédagogique. Comme a pu le dire un médecin grec au cours d'un entretien : «Le projet consiste à habituer les gens à payer jusqu'à ce que le système [de santé publique] soit finalement supprimé. » Dans sa contribution aux travaux du réseau Globalization and Health Knowledge Network, John Lister note : «Il ne fait pas de doute qu'en promouvant des frais modérateurs, la Banque mondiale et d'autres agences entendaient notamment nourrir l'apparition de polices d'assurance, même dans les pays les plus pauvres [...]. [Par exemple], à l'occasion d'un atelier majeur financé par l'Agence des États-Unis pour le développement international (USAID), qui s'est tenu au Zimbabwe, l'une des conclusions principales relatives aux "enseignements" tirés de la réflexion sur le financement des soins de santé était que "les frais modérateurs sont vitaux pour l'introduction de n'importe quel type de système assurantiel" » (McEuen et McGaugh, cité par Kondilis et al., 2013 ; Lister, 2008:34).

Tout aussi importantes ont été d'autres mesures prises en Grèce pour aller dans la même direction. La dérégulation des services privés de santé a commencé très tôt au début du premier programme d'ajustement structurel. Par exemple, Kondilis et alii (2013) mentionnent la suppression de toutes les limites à l'établissement de laboratoires, de centres médicaux et d'unités de dialyse par des entrepreneurs privés, et la levée des restrictions relatives à l'expansion des hôpitaux privés. Les auteurs relèvent également l'introduction en 2011 de contrats passés par les établissements publics avec les compagnies d'assurance privées pour leur transférer des services hospitaliers publics. Ils leur ont attribué des centaines de lits hospitaliers de luxe. Avant même la loi «Soins primaires de santé » d'Andreas Xanthos, deux grandes cliniques privées aux spécialités multiples fonctionnant en lien avec d'importants prestataires d'assurance privées avaient été créées en remplacement des structures du réseau PEDY à Athènes et à Thessalonique.

\section{Survivre sous conditions}

L'importance des déterminants sociaux de la santé pour le bien-être physique, mental et social des communautés humaines a été soulignée plus haut. Avec l'aide et une certaine complicité du gouvernement grec (Burgi, 2014a), la troïka a prétendu pallier les effets de la « crise » (les effets de la quasi-liquidation, effective ou en instance, de la protection sociale) en agissant sur ces déterminants dans l'intention de renforcer la « résilience » de la population. Les mesures phares initiées à cette fin - indemnisation et accès des chômeurs aux soins médicaux, instauration d'un revenu minimum 
garanti (RMG) - tracent, dans leur version grecque, les contours matériels et idéels du « nouveau modèle social européen » appelé à se substituer à l’État social décrété « révolu » par le gouverneur de la Banque centrale européenne, Mario Draghi, en 2012. Ce modèle est évoqué dans le mémorandum d'août 2015 qui consacre une petite section à un « authentique filet de sécurité sociale » et à " une société plus équitable ». Embrassant la notion promue par la Banque mondiale d'un « ensemble de prestations de base » (essential package) pour les plus démunis, il assure tout juste la survie des plus pauvres.

\section{III.1. La reconfiguration des indemnités de chômage et de l'assurance-maladie}

Avant juillet 2011, la durée maximale d'indemnisation du chômage, qui conditionnait l'accès aux prestations de santé, n'excédait pas un an, mais les patients en fin de droit et privés de ressources financières pouvaient encore être traités dans les hôpitaux. Après juillet 2011, de nouvelles dispositions exigèrent que les non-assurés paient intégralement leurs soins et en mars 2012, les montants de l'indemnisation furent réduits (de 561 à 360 euros ${ }^{30}$ ). Entretemps, les taux de chômage et le nombre de personnes non assurées grimpèrent en flèche et restèrent énormes jusqu’à aujourd'hui. Médecins de Monde estime qu'il y a environ 3 millions de nonassurés, si l'on inclut les auto-employés qui ne sont pas comptabilisés dans les chiffres officiels du chômage ; Makis Mantas estime la proportion de non-assurés toutes catégories confondues à $35 \%$ de la population en 2016, parmi lesquels $60 \%$ de professions indépendantes et d'auto-employés ; les rapports officiels de l'UE ou de l'OCDE avancent généralement le chiffre de 2,5 millions de non-assurés (sur une population totale de 11 millions d'habitants).

Sous la pression sociale, le ministre de la Santé, Adonis Georgiadis, introduisit par décret ministériel en 2013 puis en 2014 un système de coupons censés permettre aux non-assurés d'accéder aux soins primaires (2013) puis également aux soins hospitaliers (2014). Ces coupons, administrativement ingérables et en pratique inaccessibles au plus grand nombre, se révélèrent une mesure palliative ${ }^{31}$. Les patients se retrouvèrent dans des situations où ils devaient payer formellement ou informellement - en signant une reconnaissance de dette envers l'établissement public de soins - une partie sinon la totalité de leur traitement hospitalier, diagnostic et médicamenteux.

30. Et la durée des indemnités de chômage fut étendue pour couvrir sous de strictes conditions certaines catégories de chômeurs de longue durée en fin de droit (200 euros mensuels).

31. En colère, les médecins des cliniques sociales solidaires dénoncèrent « une goutte dans l'océan ». "Le ministre de la Santé, dirent-ils en 2013, espère nous impressionner avec une aspirine, quand une cure beaucoup plus radicale est nécessaire » (MCCH, archive 2013, http:// www.mkiellinikou.org/en/category/mcch/page/2/). 
Finalement, c'est au gouvernement Syriza qu'est revenue la responsabilité de mettre en place un plan dit d'accès « égal et universel à la santé ». Amélioration significative, la loi $\mathrm{n}^{\circ}$ 4368/16 complétée par un arrêté ministériel du 2 mars 2016 offre à tous les citoyens légalement installés en Grèce la possibilité d'être pleinement couverts sur simple présentation de leur numéro de Sécurité sociale. Les réfugiés récemment entrés et enregistrés en Grèce le sont au même titre que les autres citoyens dépourvus d'assurance sociale. Par contre, les immigrés sans papiers plus anciens, Pakistanais, Indiens ou autres, ne sont pas couverts.

Sauf pour les très pauvres, la gratuité des soins n'inclut pas les médicaments dont le coût est en moyenne supporté pour moitié par les patients. La dispense des frais modérateurs est subordonnée à des conditions telles que seul un petit nombre, estimé ${ }^{32}$ à quelque 170000 personnes, est concerné (sur au moins 2,5 millions de bénéficiaires potentiels, comme on vient de le voir). La gratuité des médicaments est soumise aux critères suivants : (1) les revenus ne doivent pas excéder 2400 euros annuels pour une personne seule (le double pour un couple avec deux enfants) ; (2) si une personne n'a pas de revenus mais possède un bien immobilier d'une valeur de 150000 euros et plus, ou si une personne a un compte en banque comprenant des avoirs équivalents à trois fois le critère annuel des 200 euros mensuels (donc un solde créditeur de 7200 euros), elle doit participer aux frais modérateurs ; (3) les handicapés dont le taux de handicap est évalué à moins de $67 \%$ ne sont pas couverts à 100 \% (avec une légère différence en leur faveur s'il y a des enfants); (4) l'accès à la consultation de spécialistes est restreint : la prestation de services publics de santé gratuits est rigoureusement limitée aux ressources publiques existantes et ne s'étend pas à des services que les hôpitaux ou les centres de santé contractent auprès de prestataires privés. Dans tous les cas où les patients habitent une contrée dépourvue du service ou du spécialiste recherchés, ils se voient obligés ou bien de voyager s'ils le peuvent pour se rendre dans une grande ville, ou bien de se passer de soins spécialisés.

\section{III.2. L'Allocation de solidarité sociale, un revenu minimum garanti}

Censé refléter l'ambition de « justice sociale » des rédacteurs du troisième mémorandum, le revenu minimum garanti (RMG) incarne bien l'idéal d'une protection sociale réduite à un plancher de prestations minimales. La Commission européenne le décrit comme un « exemple typique d'investissement social » (Ziomas et al., 2015a). L'« investissement social » est alors l'antithèse des droits (durement conquis) à des revenus de transfert inconditionnels qui sont, eux, désobligeamment appelés " consommation

32. Estimations attribuées par la presse au ministre de la Santé Andreas Xanthos et à la viceministre, Theano Fotiou. 
sociale ». La finalité de cet « investissement social » ciblé exclusivement sur la pauvreté extrême (ibid.) n'est pas de combattre l'indigence, mais de réduire l'indicateur mesurant « l'écart de pauvreté » (Ziomas et al., 2015b), c'est-à-dire l'écart relatif entre le niveau de vie médian de la population pauvre et le seuil de pauvreté.

Le RMG est entré en application en $2017^{33}$ sous l'appellation « allocation de solidarité sociale » (KEA selon l'acronyme grec). Il avait préalablement été l'objet d'une expérimentation pilote dans treize gouvernements locaux entre novembre 2014 et avril 2015. Le dispositif comprend une allocation dégressive inférieure au seuil de pauvreté extrême ${ }^{34}$ n'excédant pas 200 euros mensuels pour une personne seule, et par exemple 400 euros pour une famille de quatre personnes ou 500 euros pour un couple et quatre enfants mineurs. Il comprend également une aide sociale en nature et une aide à la recherche d'emploi. Les conditions d'attribution des aides sont analogues à celles mentionnées précédemment au sujet du programme d'accès universel à la santé. Le revenu déclaré du ménage au cours des six mois précédant la demande ne doit pas excéder six fois le montant de l'allocation ${ }^{35}$ ou un plafond fixé à 5400 euros quel que soit le nombre de personnes composant le ménage. À cela s'ajoutent des critères portant sur la propriété, qui varient aussi en fonction de la composition du ménage. Ils incluent la valeur taxable des biens immobiliers en Grèce ou à l'étranger ${ }^{36}$, le coût objectif de tous types de véhicules privés ${ }^{37}$ et le montant total des dépôts en banque ou dans toute autre institution de crédit ${ }^{38}$. La mise en œuvre du dispositif est confiée aux municipalités.

Les volets aide sociale et aide à la recherche d'emploi n'avaient pas été inclus dans l'expérimentation pilote : celui sur la recherche d'emploi parce que la Grèce est plongée dans une dépression économique durable et que les structures de ce type sont quasiment inexistantes, en tout cas inopérantes ; celui sur l'aide sociale (principalement des opérations de distribution alimentaire), parce que les conditions d'ouverture respectivement du droit au revenu minimum garanti et du droit à l'aide sociale n'étaient pas encore harmonisées. Depuis l'entrée en application du dispositif KEA, les listes de bénéficiaires du revenu minimum garanti sont utilisées par les

33. Arrêté ministériel du 24 janvier 2017 relatif à la loi $n^{\circ} 4320 / 15$.

34. Selon les résultats de l'enquête de Matsaganis et al. (2016), le seuil d'extrême pauvreté varie selon les localités considérées: Athènes, " Autres zones urbaines », "Zones rurales et périurbaines » dans leur étude. Pour une personne seule, il se situait en 2015 respectivement à 222, 216 et 182 euros ; pour un couple avec deux enfants : 640, 614 et 524 euros. Ces chiffres concernent la population qui ne doit payer ni loyer ni emprunt immobilier. Avec un loyer ou un emprunt immobilier, les seuils sont plus élevés.

35. 1200 euros pour une personne seule, un peu plus selon la composition du ménage, par exemple 3000 euros pour un couple et quatre enfants.

36.90000 euros pour une personne seule, avec un plafond fixé à 150000 euros.

37. Voitures, et même bicyclettes : il ne doit pas excéder 6000 euros.

38.4800 euros pour une personne seule, 9600 euros pour deux adultes et deux enfants mineurs, 14400 euros pour deux adultes et six enfants mineurs, avec des plafonds intermédiaires renvoyant à la composition des ménages. 
services sociaux municipaux pour l'attribution d'une aide sociale et sont parfois croisées avec des listes établies par d'autres structures, dont les églises, pour éviter que le même individu puisse profiter simultanément de plusieurs programmes d'aide. À partir de début 2018 aura lieu le renouvellement du droit à l'allocation KEA de solidarité sociale. À cette occasion, les bénéficiaires admis en 2017 qui n’auraient pas su qu'ils devaient, ou n'auraient pas voulu déclarer une éventuelle modification de leur situation dans le cours de l'année, ou ceux dont on s'apercevra que le dossier déposé en 2017 comporte des erreurs, quelles qu'elles soient et même si c'est à leur insu ${ }^{39}$, seront sommés de rembourser les sommes qui leur ont été versées ${ }^{40}$. On ne sait pas ce qu'il adviendra de ces bénéficiaires ni comment le problème sera géré. En tout état de cause, l'adjonction de l'aide sociale au revenu minimum n'est pas de nature à consolider les déterminants sociaux de la santé.

\section{III.3. Le cadre de la survie}

De ce qui précède, il est permis de penser que l'allocation de solidarité sociale, composante centrale de ce qui est présenté comme un filet social, est appelée à devenir un dispositif intégré de coordination de politiques dites de protection sociale ciblées sur la misère, qui produisent, normalisent et contrôlent le statut de dépossédé ; un dispositif de gestion limité aux $15 \%$ de la population classés comme extrêmement pauvres. Les conséquences sociales des politiques austéritaires se réduiraient à la « résilience » défectueuse d'une minorité.

Notion polysémique, la résilience sociale peut s'entendre comme la capacité des individus ou des groupes de résister à l'adversité en mobilisant et en inventant de nouvelles ressources et manières de faire et d'agir pour préserver leur bien-être matériel, physique et psychique, ainsi que la dignité conférée par la reconnaissance de soi et d'autrui comme membre à part entière d'une communauté de semblables. Bien sûr, les réponses individuelles et collectives à la déstabilisation des cadres sociaux diffèrent sensiblement d'une société à l'autre (Hall, Lamont, 2013). Pour ce qui concerne la santé publique en Grèce, la création, évoquée plus loin, d’un réseau de structures de soins solidaires dans tout le pays a été portée par l'espoir de préserver la substance de la société et défendre ses droits.

39. Les demandeurs ont la possibilité de faire leur demande en ligne soit par eux-mêmes, soit avec l'aide d'un comptable, soit en passant par un centre de services aux citoyens (KEP), soit directement à la mairie. II semble que les comptables et les KEP, peu familiers de la procédure, aient mal questionné ou informé les demandeurs et involontairement omis de mentionner des détails relatifs à leurs obligations et à leur situation (sources de revenus ou autres). Or, les demandeurs doivent signer un formulaire attestant de la véracité de la déclaration, et sont considérés comme responsables en cas d'inexactitude.

40. Entretiens effectués en octobre 2017 dans les municipalités portuaires de Perama et KeratsiniDrapetsona dans le cadre d'une enquête qualitative en cours effectuée par l'auteure de ces lignes avec l'historienne Eleni Kyramargiou, chercheure à la Fondation nationale de la recherche historique en Grèce. 
Ce n'est pas ainsi que les institutions dominantes conçoivent la notion de résilience. Elles donnent à ce vocable un sens normatif inférant une soumission allant de soi à l'ordre établi. Dans son acception politicoadministrative, la résilience désigne la résistance avant tout psychologique des individus à l'infortune (euphémisme pour injustice) au sens de leur aptitude à réaliser un " ajustement positif " pour s'adapter et « rebondir » dans les contextes les plus difficiles - qui ne sont pas, eux, questionnés (par exemple Burgi, Soumara, 2015). Cette grammaire de la résilience induit la conviction qu'il faut s'accommoder de la (grande) précarité, contrôler le " désordre », les comportements " antisociaux », les troubles à l'ordre public, et légitimer si nécessaire des mesures d'exception et/ou le recours à la force. Il appartiendrait à chacun et à tous de se prendre en charge. Seules les catégories sociales totalement démunies (à l'exclusion des réfugiés et autres immigrés) pourraient espérer, sous d'austères conditions, une aide matérielle parcimonieuse financée par la communauté.

La troïka trouve dans ces préceptes un moyen parmi d'autres de passer sous silence sinon de nier les conséquences de ses politiques. Avant la crise financière, les gens allaient chez le médecin malgré les carences des soins primaires. Maintenant, ils doivent se résoudre à une mort à échéance plus ou moins précoce. Même les assurés sociaux, souvent incapables de couvrir les dépenses non remboursables, ont tendance à négliger les examens préventifs, à les reporter à plus tard (ou trop tard) ; ils réduisent leurs traitements, les prennent de façon discontinue, les remplacent par des substituts moins chers, les arrêtent même. Des maladies que l'on croyait éradiquées comme la malaria réapparaissent, le VIH s'est répandu. L'état de la santé mentale est désastreux. Dès le premier mémorandum, les médecins hospitaliers constataient une augmentation des pathologies cardiaques et accidents vasculaires cérébraux liés à l'anxiété et au stress ${ }^{41}$. Le taux de dépressions majeures est passé entre 2009 et 2014 de 3,5\% à 12,5\% de la population et le taux de suicides s'est accru de $35 \%$ entre 2010 et 2013 (Economou, Peppou, Fousketatki et al., 2013 ; Economou, Madianos, Peppou et al., 2013 ; Economou et al., 2016 ; Madianos et al., 2014). La consommation de «drogues de la crise »- substances synthétiques très bon marché comme la métamphétamine ( 1 à 5 euros) fabriquées dans des laboratoires de fortune et même dans les cuisines des particuliers - pourrait détruire une génération entière d'adolescents, comme le sisa en Argentine (Matsa, 2014). La désintégration de la société et le désastre sanitaire nourrissent la violence interpersonnelle (homicides et violence domestique). Le psychiatre Spyros Sourlas (entretien, 2015) a observé un accroissement de $30 \%$ des troubles psychosomatiques (maux de tête, de ventre) parmi les enfants, dont un tiers finit à l'hôpital. Gerasimos Kolaitis et Georges Giannakopoulos (2015),

41. Entretiens à l'hôpital universitaire de Thessalonique, 2011. 
tous deux pratiquant dans d'importants services hospitaliers de psychiatrie infantile à Athènes, rapportent qu'ils rencontrent un « nombre toujours grandissant de familles confrontées à des adversités psychosociales compliquées » et d'enfants maltraités ou négligés admis dans le plus grand hôpital pédiatrique de Grèce au titre de la protection de l'enfance...

Les traités font obligation à la Commission européenne d'évaluer les effets sociaux et sanitaires de toutes les politiques publiques, y compris celles de la troïka. Elle y a rechigné. Une étude d'impact social a fini par être réalisée a posteriori en 2015, mais elle fut pour le moins « décevante à bien des égards ", comme le note dans sa déclaration de fin de mission sur l'état social et humain de la Grèce Juan Pablo Bohoslavsky (2015), expert indépendant sur la dette extérieure et les droits humains auprès des Nations unies. Elle «ne mentionne pas une seule fois la notion de "droits humains" » fondamentaux, poursuit l'expert, dont le rapport pointe au contraire une violation systématique de ces droits ${ }^{42}$. La « vertigineuse liste de normes, règles, lois grecques, européennes et internationales piétinées par les mémorandums » (CADTM, 2015) est aujourd'hui assez bien répertoriée (par exemple Salomon, 2015 ; Ghailani, 2016) mais mal connue du grand public mieux informé sur l'interprétation dominante des abondantes données économiques et financières que sur les atteintes à la démocratie.

Pour leur part, les gouvernements grecs se sont montrés tout aussi cyniques et indifférents au sort de la population. Ils ont par exemple reporté sur les femmes et les migrants la responsabilité de la crise sanitaire. En 2012 et 2013, ils orchestrèrent des campagnes de « nettoyage » contre les consommateurs de drogue et les migrants. Ils mirent le pays en garde contre la propagation du Sida - qui « peut être transmis par une femme migrante illégale au consommateur grec, à la famille grecque » - et contre les «bombes à retardement sanitaires » menaçant les hommes grecs et les ménages. Un décret (39A) de 2012 permettant à la police de détenir n'importe qui en vue de procéder à des tests forcés de dépistage des maladies infectieuses et de rendre publiques les données personnelles des sujets atteints du VIH entraîna de multiples rafles, des poursuites pénales, et l'arrestation, l'emprisonnement, la stigmatisation et l'humiliation de milliers de personnes (Vasilopoulou, 2014:225-227). Il fut définitivement abrogé en avril 2015 par le gouvernement Syriza, à qui fut cependant transférée par les puissances dominantes de l'UE la charge et la responsabilité de « traiter » au moins 60000 réfugiés actuellement bloqués en Grèce où ils sont confrontés à la politique de détention automatique dans des camps inhumains et aux plus grandes difficultés d'accès (si tant est qu'ils accèdent) à des soins médicaux essentiels. Alors qu'il n’y a pas de relation de cause

42. C. Filoni, « Résumé du Rapport de l'expert de l'ONU sur la dette sur la Grèce », CATDM, 18 juillet 2014, http://www.cadtm.org/Resume-du-Rapport-de-I-expert-de-I. 
à effet entre la migration et l'importation de maladies contagieuses (par exemple Langlois et al., 2016 ; Rechel et al., 2011 ; Grove, Zwi, 2006), ces traitements spéciaux pourraient bien rendre encore plus critique la crise sanitaire et finir par mettre en danger la société tout entière.

\section{Conclusion}

Loin de cette logique mortifère, une partie de la société a pris un chemin d'une tout autre nature pour défendre la santé publique et ses conditions de possibilité. Dans un élan de solidarité remarquable, des hommes et des femmes bénévoles, médecins, soignants ou simples citoyens ont œuvré pour créer et faire fonctionner à partir de 2009 une quarantaine de cliniques et pharmacies autogérées dans tout le pays. Toute personne nécessitant des soins médicaux sans y avoir accès pour une raison ou une autre y est accueillie indépendamment de sa nationalité, de son statut social ou de ses origines. Ces structures ont sauvé des milliers de vies, mais leurs maigres ressources, provenant exclusivement de dons de la population et de réseaux militants nationaux et internationaux (dont le collectif Solidarité France-Grèce pour la Santé) ne leur permettent de répondre qu'à une part minime des besoins de la population. La lutte ainsi engagée au nom de la santé pour tous n'a pas eu pour objet de construire une alternative au système public de santé. Elle a été pensée, menée et vécue comme un acte de résistance positive contre un pouvoir despotique, un acte susceptible d'amener les habitants à se relever et défendre par leurs pratiques solidaires leurs droits et les principes démocratiques. Cet espoir s'essouffle depuis que le gouvernement Syriza et son Premier ministre, choisissant de conserver le pouvoir après la capitulation d'Alexis Tsipras en juillet 2015, appliquent un troisième programme mémorandaire plus violent et punitif encore que les deux précédents. L'apathie gagne du terrain. Les militants et bénévoles, épuisés, quittent les organisations de solidarité. Nul ne sait par quels moyens la société grecque cherchera dans le dénouement de cette tragédie à se défendre contre les atteintes portées à sa substance même. 


\section{Références bibliographiques}

Anastasaki E., Bradshaw S., Proach J., Shah S. (2014), « The Greek Healthcare Reform After Troika: The Potential Impact on Global Pricing and Access Strategy ", Presented at the International Health Economics and Outcomes Research (ISPOR), 17th Annual European Congress, Amsterdam, https:// www.ispor.org/research_pdfs/48/pdffiles/PHP152.pdf.

Bohoslavsky J.P. (2015), " End of Mission Statement », United Nations, Human Rights - Office of the High Commissioner, Athens, December 8, http://www.ohchr.org/Documents/Issues/IEDebt/EOM_Statement_Greece_ IEForeignDebt_EN.pdf.

Burgi N. (2014a), « Introduction », in Burgi N. (dir.), La grande régression : la Grèce et l'avenir de l'Europe, Lormont, Le Bord de l'eau, p. 15-52.

Burgi N. (2014b), « Démantèlement de la santé, destruction de la société », in Burgi N. (dir.), La grande régression : la Grèce et l'avenir de l'Europe, Lormont, Le Bord de l'eau, p. 193-212.

Burgi N. (dir.) (2014c), La grande régression : la Grèce et l'avenir de l'Europe, Lormont, Le Bord de l'eau.

Burgi N., Soumara G. (2015), "Que disent les enfants de la crise humanitaire en Grèce ? ", Tepsis Paper, https://hal.archives-ouvertes.fr/hal-01192822/ document.

CADTM (2015). Rapport préliminaire de la Commission pour la vérité sur la dette publique grecque, 24 juillet, http://www.cadtm.org/IMG/pdf/Raport_FR_2307.pdf.

Carone G., Schwierz C., Xavier A. (2012), « Cost-Containment Policies in Public Pharmaceutical Spending in the EU », European Economy Economic Papers, $\mathrm{n}^{\circ}$ 461, European Commission - Directorate-General for Economic and Financial Affairs, Brussels, September, http://ec.europa.eu/ economy_finance/publications/economic_paper/2012/pdf/ecp_461_en.pdf.

Commission on Social Determinants of Health (CSDH) (2008), Closing the Gap in a Generation: Health Equity Through Action on the Social Determinants of Health. Final Report of the Commission on Social Determinants of Health, Geneva, World Health Organization., http://apps.who.int/iris/bitstre am/10665/43943/1/9789241563703_eng.pdf

Correia T., Dussault G., Pontes C. (2015), « The Impact of the Financial Crisis on Human Resources for Health Policies in Three Southern-Europe Countries ", Health Policy, vol. 119, $\mathrm{n}^{\circ}$ 12, p. 1600-1605, https://doi.org/10.1016/j. healthpol.2015.08.009.

Daniels N., Kennedy B.P., Kawachi I. (1999), « Why Justice Is Good for Our Health: The Social Determinants of Health Inequalities ", Daedalus, vol. 128, $n^{\circ} 4$, p. 215-251, http://www.jstor.org/stable/20027594?seq=1\#page_scan_tab_ contents.

Economou M., Angelopoulos E., Peppou L.E., Souliotis K., Stefanis C. (2016), " Suicidal Ideation and Suicide Attempts in Greece During the Economic Crisis: An Update », World Psychiatry, vol. 15, n 1, p. 83-84, https://doi. org/10.1002/wps.20296.

Economou M., Madianos M., Peppou L.E., Theleritis C., Patelakis A., Stefanis C. (2013), "Suicidal Ideation and Reported Suicide Attempts in Greece 
During the Economic Crisis », World Psychiatry, vol. 12, $\mathrm{n}^{\circ} 1$, p. 53-59, https://doi.org/10.1002/wps.20016.

Economou M., Peppou L., Fousketaki S., Theleritis C., Patelakis A., Alexiou T., Madianos M., Stefanis C. (2013), « Economic Crisis and Mental Health: Effects on the Prevalence of Common Mental Disorders », Psychiatriki, vol. $24, n^{\circ} 4$, p. $247-261$.

European Commission (2010), « The Economic Adjustement Programme for Greece ", Occasional Papers, $n^{\circ}$ 61, Directorate-General for Economic and Financial Affairs, Brussels, May, http://ec.europa.eu/economy_finance/ publications/occasional_paper/2010/pdf/ocp61_en.pdf.

European Commission (2012), «The Second Economic Adjustment Programme for Greece », Occasional Papers, $n^{\circ}$ 94, Directorate-General for Economic and Financial Affairs, Brussels, March, http://ec.europa.eu/economy_finance/ publications/occasional_paper/2012/pdf/ocp94_en.pdf.

Fonds monétaire international (2012), Perspectives de l'économie mondiale, octobre 2012. Une dette élevée et une croissance anémique, Fonds monétaire international.

Foucault M. (2004), Naissance de la biopolitique : cours au Collège de France, 1978-1979, Paris, Gallimard.

Gandjour A. (2013), « Reference Pricing and Price Negotiations for Innovative New Drugs: Viable Policies in the Long Term? ॥, PharmacoEconomics, vol. 31, $\mathrm{n}^{\circ}$ 1, p. 11-14, https://doi.org/10.1007/s40273-012-0002-9.

Georgakopoulos T. (2016), " The Health of Greeks and the Crisis: A Study. Dianeosis, http://www.dianeosis.org/2016/03/greek_health_intro/

Ghailani, D. (2016). «Violations of Fundamental Rights: Collateral Damage of the Eurozone Crisis? », OSE Research Paper, n³2, December, http://www. ose.be/files/publication/OSEPaperSeries/Ghailani_2016_OseResearchPaper32EN.pdf.

Greer S.L., Jarman H., Baeten R. (2016), « The New Political Economy of Health Care in the European Union: The Impact of Fiscal Governance », International Journal of Health Services, vol. 46, $\mathrm{n}^{\circ} 2$, p. 262-282, https://doi. org/10.1177/0020731416637205.

Grimaldi A. (2009), L’hôpital malade de la rentabilité, Paris, Fayard.

Grove N.J., Zwi A.B. (2006), « Our Health and Theirs: Forced Migration, Othering, and Public Health », Social Science \& Medicine, vol. 62, n 8, p. 1931-1942, https://doi.org/10.1016/j.socscimed.2005.08.061.

Hall P.A., Lamont M. (eds.) (2013), Social Resilience in the Neoliberal Era, Cambridge, Cambridge University Press.

Hayek F.A. von (1985), La route de la servitude, Paris, Puf.

Herndon T., Ash M., Pollin R. (2013), « Does High Public Debt Consistently Stifle Economic Growth? A Critique of Reinhart and Rogoff », Working Paper Series, $\mathrm{n}^{\circ}$ 322, Political Economy Research Institute, University of Massachusetts, April 15, http://darribas.org/WooWii/Assignments/OriginalPaper.pdf.

Ifanti A.A., Argyriou A.A., Kalofonou F.H., Kalofonos H.P. (2014), « Physicians' Brain Drain in Greece: A Perspective on the Reasons Why and How to Address It », Health Policy, vol. 117, $\mathrm{n}^{\circ}$ 2, p. 210-215, https://doi.org/10.1016/j. healthpol.2014.03.014. 


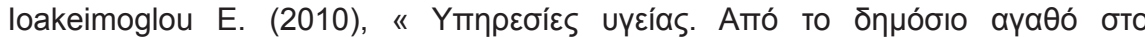
$\varepsilon \mu т о ́ p \varepsilon u \mu \alpha$ ", ("Services de santé. Du bien public à la marchandise »), Meletes, $\mathrm{n}^{\circ} 32$, Athènes, Institut du Travail INE/GSEE-ADEDY, http://www. inegsee.gr/wp-content/uploads/2014/02/files/MELETH\%2032\%20TELIKO. pdf.

loakeimoglou E. (2017), " Internal Devaluation and Hegemonic Crisis ", in Fouskas V.K., Dimoulas C. (eds.), Greece in the $21^{\text {st }}$ Century: A Crisis Manifesto, London, Routledge, forthcoming.

Jayadev A., Konczal M. (2010), " The Boom Not the Slump: The Right Time for Austerity », Economics Faculty Publication Series, Paper 26, University of Massachusetts Boston/Roosevelt Institute, August 23.

Kaitelidou D., Kouli E. (2012), « Greece: The Health System in a Time of Crisis », Eurohealth Incorporating Euro Observer, vol. 18, $\mathrm{n}^{\circ} 1$, p. 12-14, http:// www.euro.who.int/_data/assets/pdf_file/0005/162959/Eurohealth_Vol-18_ No-1_web.pdf.

Kapsalis A., Kouzis Y. (2014), " Le travail, la crise et les mémorandums », in Burgi N. (dir.), La grande régression: la Grèce et l'avenir de l'Europe, Lormont, Le Bord de l'eau, p. 156-174.

Karakioulafis C. (2014), "Grèce : santé et éducation : victimes des politiques d'austérité ", $\mathrm{n}^{\circ}$ spécial, "Santé, éducation : services publics dans la tourmente ", Chronique Internationale de I'IRES, $n^{\circ} 148$, novembre, p. 83103, http://www.ires.fr/publications/chronique-internationale-de-l-ires/ item/3556-grece-sante-et-education-victimes-des-politiques-d-austerite.

Karamanoli E. (2015), « 5 Years of Austerity Takes its Toll on Greek Health Care », The Lancet, vol. 386, $\mathrm{n}^{\circ}$ 10010, December 5, p. 2239-2240, https://doi. org/10.1016/S0140-6736(15)01163-0.

Karanikolos M., Mladovsky P., Cylus J., Thomson S., Basu S., Stuckler D., Mackenbach J.P., McKee M. (2013), « Financial Crisis, Austerity, and Health in Europe ", The Lancet, vol. 381, n 9874, April 13, p. 1323-1331, https:// doi.org/10.1016/S0140-6736(13)60102-6.

Kentikelenis A., Karanikolos M., Reeves A., McKee M., Stuckler D. (2014), « Greece's Health Crisis: From Austerity to Denialism », The Lancet, vol. 383, n 9918 , February 22, p. 748-753, https://doi.org/10.1016/S0140-6736(13)62291-6.

Kondilis E., Giannakopoulos S., Gavana M., lerodiakonou I., Waitzkin H., Benos A. (2013), «Economic Crisis, Restrictive Policies, and the Population's Health and Health Care: The Greek Case ", American Journal of Public Health, vol. 103, $\mathrm{n}^{\circ}$ 6, April, p. 973-979, https://doi.org/10.2105/ AJPH.2012.301126.

Kondilis E., Smyrnakis E., Gavana M., Giannakopoulos S., Zdoukos T., Iliffe S., Benos A. (2012), " Economic Crisis and Primary Care Reform in Greece: Driving the Wrong Way? », British Journal of General Practice, vol. 62, n 598, May, p. 264-265, https://doi.org/10.3399/bjgp12X641546.

Kotronaki L. (2014), « Réapproprier la contestation démocratique: la forme Occupy », in Burgi N. (dir.), La grande régression : la Grèce et l'avenir de l'Europe, Lormont, Le Bord de l'eau, p. 175-190.

Laliotis I., Ioannidis J.P.A., Stavropoulou C. (2016), « Total and Cause-specific Mortality Before and After the Onset of the Greek Economic Crisis: An Interrupted Time-series Analysis ", The Lancet Public Health, vol. 1, $\mathrm{n}^{\circ} 2$, December, p. e56-e65, https://doi.org/10.1016/S2468-2667(16)30018-4. 
Langlois E.V., Haines A., Tomson G., Ghaffar A. (2016), « Refugees: Towards Better Access to Health-care Services ", The Lancet, vol. 387, $\mathrm{n}^{\circ} 10016$, January 23, p. 319-321, https://doi.org/10.1016/S0140-6736(16)00101-X.

Lister J. (2008), « Globalization and Health Systems Change », Globalization and Health Knowledge Network: Research Papers, WHO Commission on Social Determinants of Health, Institute of Population Health, Ottawa, http://www. globalhealthequity.ca/webfm_send/14.

Lochard Y., Pernot J.-M. (2010), « Europe : 2010, année terrible. Les relations sociales à l'épreuve de l'austérité », $\mathrm{n}^{\circ}$ spécial, "L'État social à l'épreuve de l'austérité ॥, Chronique internationale de I'IRES, $\mathrm{n}^{\circ}$ 127, novembre, p. 40-55, http://www.ires.fr/publications/chronique-internationale-de-l-ires/ item/3668-europe-2010-annee-terrible-les-relations-sociales-a-l-epreuvede-l-austerite.

Madianos M.G., Alexiou T., Patelakis A., Economou M. (2014), « Suicide, Unemployment and Other Socioeconomic Factors: Evidence from the Economic Crisis in Greece », The European Journal of Psychiatry, vol. 28, n 1, p. 3949, https://doi.org/10.4321/S0213-61632014000100004.

Matsa K. (2014), " Les addictions en temps de crise », in Burgi N. (dir.), La grande régression: la Grèce et l'avenir de l'Europe, Lormont, Le Bord de l'eau, p. $213-230$.

Matsaganis M., Leventi C. (2013), « Anatomy of Poverty in 2013 Greece », Newsletter, $\mathrm{n}^{\circ} 5$, Athens University of Economics, Public Policy Research Unit, http://www.paru.gr/files/newsletters/NewsLetter_05.pdf.

Matsaganis M., Leventi C., Flevotomou M. (2016), « Une politique plus efficace pour combattre l'extrême pauvreté », Dianeosis, juillet, http://www.dianeosis.org/wp-content/uploads/2016/06/ftwxeia_version_070616_3.pdf.

Mossialos E., Allin S., Davaki K. (2005), « Analysing the Greek Health System: A Tale of Fragmentation and Inertia ", Health Economics, vol. 14, $\mathrm{n}^{\circ} \mathrm{S} 1$, September, p. S151-S168, https://doi.org/10.1002/hec.1033.

OCDE (2015), Réformes économiques 2015. Objectif croissance, Paris, Éditions de l'OCDE, https://doi.org/10.1787/growth-2015-fr.

Parlement hellénique, Bureau du Budget de l'État (2014), Politiques de revenu minimum dans l'UE et en Grèce. Une analyse comparative, Document préparatoire du programme pilote de revenu minimum garanti, Rapport intermédiaire aux membres des commissions parlementaires compétentes, Athènes, septembre.

Rechel B., Mladovsky P., Devillé W., Rijks B., Petrova-Benedict R., McKee M. (2011), " Migration and Health in the European Union: An Introduction ", in Rechel B., Mladovsky P., Devillé W., Rijks B., Petrova-Benedict R., McKee M. (eds.), Migration and Health in the European Union, Maidenhead, Mc Graw-Hill, Open University Press, p. 3-13, http://www.mheducation. co.uk/openup/chapters/9780335245673.pdf.

Sachs J.D. (2005), Investing in Development: A Practical Plan to Achieve the Millennium Development Goals, London, Sterling, Earthscan, http://www.unmillenniumproject.org/documents/MainReportComplete-lowres.pdf.

Salomon M.E. (2015), "Of Austerity, Human Rights and International Institutions », LSE Law, Society and Economy Working Papers, n² 2/2015, http://www.lse. ac.uk/humanRights/documents/2015/salomonWpsAusterity.pdf. 
Stamati F., Baeten R. (2014), " Health Care Reforms and the Crisis », ETUI Report, $n^{\circ}$ 134, Brussels http://www.etui.org/Publications2/Reports.

Stuckler D., Basu S. (2014), Quand l'austérité tue. Épidémies, dépressions, suicides : l'économie inhumaine, Paris, Autrement ; traduit de Stuckler D., Basu S. (2013), The Body Economic: Why Austerity Kills: Recessions, Budget Battles, and the Politics of Life and Death, New York, Basic Books.

Triantafyllou K., Angeletopoulou C. (2011), « IMF and European Co-workers Attack Public Health in Greece », The Lancet, vol. 378, n 9801, October, p. 14591460, https://doi.org/10.1016/S0140-6736(11)61639-5.

Vasilopoulou C. (2014), « L'emprisonnement des prostituées séropositives stigmatisées et la création d'un camp de détention pour sans-papiers et autres exclus ", in Burgi N. (dir.), La grande régression: la Grèce et l'avenir de l'Europe, Lormont, Le Bord de l'eau, p. 225-227.

Ziomas D., Sakellis I., Bouzas N., Spyropoulou N. (2015a), ESPN Thematic Report on Minimum Income Schemes: Greece 2015, European Commission, Directorate-General for Employment, Social Affairs and Inclusion, Brussels.

Ziomas D., Sakellis I., Bouzas N., Spyropoulou N. (2015b), ESPN Thematic Report on Social Investment. Greece, European Commission, Directorate-General for Employment, Social Affairs and Inclusion, Brussels. 